\title{
Impact of Variances of Random Weights and Biases on Extreme Learning Machine
}

\author{
Xiao $\mathrm{Tao}^{1}, \mathrm{Xu} \mathrm{Zhou}^{2}$, Yu Lin $\mathrm{He}^{3 *}$, Rana Aamir Raza Ashfaq ${ }^{4}$ \\ ${ }^{1}$ College of Science, Agricultural University of Hebei, Baoding 071001, China. \\ 2 Department of Basic Courses, Agricultural University of Hebei, Huanghua 061100, China. \\ ${ }^{3}$ College of Computer Science \& Software Engineering, Shenzhen University, Shenzhen 518060, China. \\ ${ }^{4}$ Department of Computer Science, Bahauddin Zakariya University, Multan, Pakistan. \\ * Corresponding author. Tel.: +86-18531315747; email: csylhe@126.com, yulinhe@szu.edu.cn \\ Manuscript submitted December 9, 2015; accepted March 12, 2016. \\ doi: 10.17706/jsw.11.5.440-454
}

\begin{abstract}
Although the uniform convergence of extreme learning machine (ELM) has been proved for any continues probability distribution, the variances of random numbers initializing input-layer weights and hidden-layer biases indeed have the obvious impact on generalization performance of ELM. In this paper, we validate this effect by testing the classification accuracies of ELMs initialized by the random numbers with different variances. We select three commonly-used probability distributions (i.e., Uniform, Gamma and Normal) and $30 \mathrm{UCI}$ data sets to conduct our comparative study. The experimental results present some important and valuable observations and instructions: (1) Uniform and Gamma distributions with the smaller variances usually make ELMs get the higher training and testing accuracies; (2) In comparison with Normal distribution, the variances of Uniform and Gamma distributions have the significant impact on classification performance of ELMs; (3) Uniform and Gamma distributions with the larger variances could seriously degrade the classification capability of ELMs; (4) ELMs initialized by Uniform and Gamma distributions with the larger variances generally needs the more hidden-layer nodes to achieve the equivalent classification accuracies with ones having the smaller variances; and (5) Normal distribution are more easily lead to the over-fitting of ELMs.
\end{abstract}

Key words: Extreme learning machine, random initialization, probability distribution, variance.

\section{Introduction}

Extreme learning machine (ELM) [1] is a special Single hidden-Layer Feed-forward neural Network (SLFN) in which the input-layer weights and hidden-layer biases are randomly selected and the output-layer weights are analytically determined by solving Moore-Penrose generalized inverse [2] of hidden-layer output matrix. Because of avoiding the time-consuming iterations, ELMs obtain the extremely fast training speed. Meanwhile, the theoretically proof [3] guarantees the universal approximate capability of ELM. The lower computational complexity and acceptable generalization performance make ELM obtain a wide range of practical applications [4].

However, ELM has an obvious defect, i.e., the instability of predictive results. Although Huang et al. in [1] proved the uniform convergence of ELM for any continuous probability distribution, this defect indeed exists in the practical applications due to the inherent character of data set, e.g., noise and outlier, etc. The researchers have developed some representative works to improve the instability of ELM caused by random 
initializations of input-layer weights and hidden-layer biases. Zhu et al. [5] proposed an Evolutionary ELM (E-ELM) which uses the differential evolutionary algorithm to select the input weights and hidden biases for ELM. Then, Cao et al. [6] improved E-ELM and developed a Self-adaptive E-ELM (SaE-ELM) to optimize the necessary parameters. Experimental results show that SaE-ELM outperforms E-ELM. An Optimized ELM (O-ELM) was designed by Matias et al. [7], which used three different optimization algorithms to optimize the input-later weights, hidden-layer biases, and regularization factor simultaneously. Heeswijk et al. [8] proposed two weight initialization schemes, i.e., binary ELM based on $\{0,1\}$-weights and ternary ELM based on $\{-1,0,1\}$-weights, to improve the diversity of neurons in the hidden layer. For binary/ternary ELMs, the necessary optimizations are also required to select the better parameters. Form the above-mentioned description, we can find that E-ELM, SaE-ELM, O-ELM, and binary/ternary ELMs used the different optimization algorithms to tune the initially random input-layer weights and hidden-layer biases. These works confirmed the impact of random initialization on generalization capability of ELM.

The Uniform random numbers in interval $[0,1]$ are commonly used to initialize the input-layer weights and hidden-layer biases, including ELM [1], E-ELM [5], SaE-ELM [6], O-ELM [7], binary/ternary ELMs [8], and other forms of improved ELM [3], [9]-[15]. Then, are Uniform random numbers in the larger intervals also suitable to initialize ELM? The main objective of this paper is to validate the impact of range of random numbers on generalization performance of ELM. Specifically, we want to clarify the influence of variances of random weights and biases on the training and testing accuracies of ELM. Three mostly-used continuous probability distributions [16], i.e., Uniform, Gamma and Normal, are employed in our study to initialize the input-layer weights and hidden-layer biases for ELMs. For each distribution, 6 different parameter pairs are initialized as shown in Fig. 1, which can be used to control the variance of random numbers. We select 30 UCI classification data sets which involve a wide range of real domains and data characteristics. In our experiment, the 10 -fold cross-validation procedure is repeated 100 times. In every time of 10 -fold cross-validation, the different ELMs are trained on the same training sets and evaluated on the same testing set. The training and testing accuracies on given data set are the average values of 100 times of 10 -fold cross-validations. We further use Wilcoxon signed-ranks test and Friedman test [17] to compare the classification performances of different ELMs on 30 data sets. The experimental results and statistical analysis show that the variance of random weights and biases indeed have the obvious impact on the generalization performance of ELM. The obtained conclusions from experimental results can instruct users choose the appropriate weights and biases for ELM so as to reduce the possibility of instability of ELM caused by random initializations.

The rest of the paper is organized as follows: In Section 2, we summarize the basic extreme learning machine. In Section 3, we experimentally validate the influence of initializations obeying different probability distributions on generalization performance of ELM. We conclude this work with some remarks in the last section.

\section{Extreme Learning Machine}

Here, we consider using extreme learning machine (ELM) [1] to deal with the classification problem based on the training dataset $\left(X_{N \times D}, Y_{N \times C}\right)$ and testing dataset $\left(X_{M \times D}^{\prime}, Y_{M \times C}^{\prime}\right)$, where

$$
X_{N \times D}=\left[\begin{array}{c}
x_{1} \\
\vdots \\
x_{N}
\end{array}\right]=\left[\begin{array}{ccc}
x_{11} & \cdots & x_{1 D} \\
\vdots & \ddots & \vdots \\
x_{N 1} & \cdots & x_{N D}
\end{array}\right], Y_{N \times C}=\left[\begin{array}{c}
y_{1} \\
\vdots \\
y_{N}
\end{array}\right]=\left[\begin{array}{ccc}
y_{11} & \cdots & y_{1 C} \\
\vdots & \ddots & \vdots \\
y_{N 1} & \cdots & y_{N C}
\end{array}\right] \text {, }
$$




$$
\mathrm{X}_{M \times D}^{\prime}=\left[\begin{array}{c}
\mathrm{x}_{1}^{\prime} \\
\vdots \\
\mathrm{x}_{M}^{\prime}
\end{array}\right]=\left[\begin{array}{ccc}
x_{11}^{\prime} & \cdots & x_{1 D}^{\prime} \\
\vdots & \ddots & \vdots \\
x_{M 1}^{\prime} & \cdots & x_{M D}^{\prime}
\end{array}\right], Y_{M \times C}^{\prime}=\left[\begin{array}{c}
y_{1}^{\prime} \\
\vdots \\
y_{M}^{\prime}
\end{array}\right]=\left[\begin{array}{ccc}
y_{11}^{\prime} & \cdots & y_{1 C}^{\prime} \\
\vdots & \ddots & \vdots \\
y_{M 1}^{\prime} & \cdots & y_{M C}^{\prime}
\end{array}\right],
$$

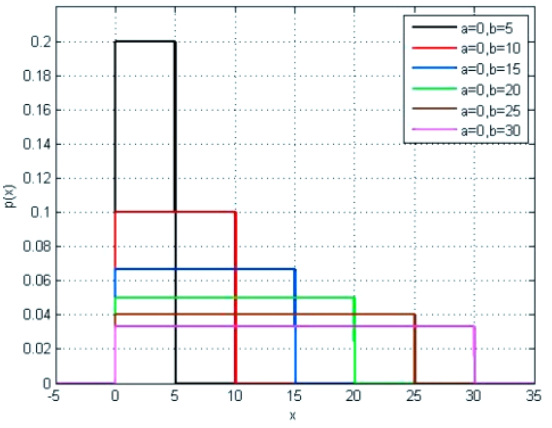

(a) Uniform distributions

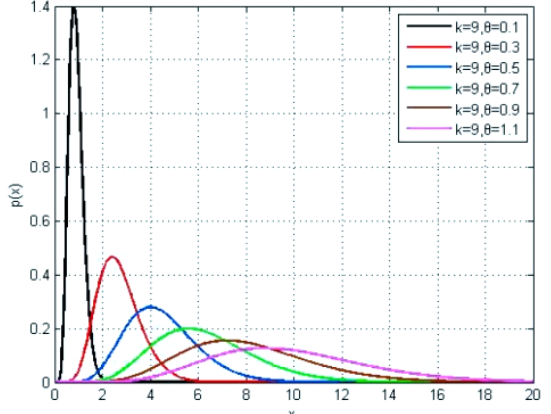

(b) Gamma distributions

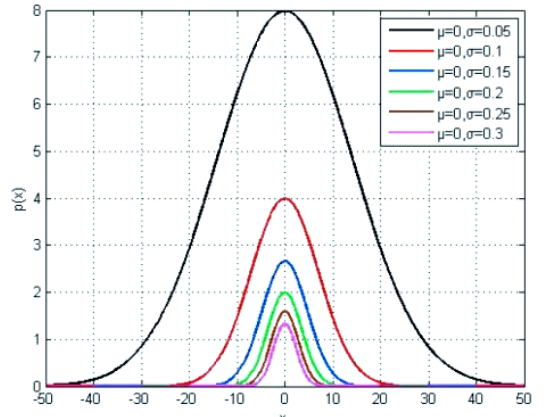

(c) Normal distributions

Fig. 1. Three continuous probability distributions for ELM initialization: for Uniform distribution, its probability density function (p.d.f.) is $p(x)=\frac{1}{b-a}, x \in[a, b]$ and variance is $\frac{(b-a)^{2}}{12}$; for Gamma, its p.d.f. is $p(x)=x^{k-1} \frac{e^{-\frac{x}{\theta}}}{\theta^{k} \Gamma(k)}, k>0, \theta>0, x \in[0,+\infty)$ and variance is $k \theta^{2}$, where $\Gamma(k)=\int_{0}^{+\infty} v^{k-1} e^{-v} d v$ is Gamma function; for Normal, its p.d.f. is $p(x)=\frac{1}{\sqrt{2 \pi} \sigma} e^{-\frac{(x-\mu)^{2}}{2 \sigma^{2}}}, \mu \in R, \sigma^{2}>0, x \in(-\infty,+\infty)$ and variance is $\sigma^{2}$.

$N$ is the number of training instances, $D$ is the number of input variables, $C$ is the number of output variables, and $M$ is the number of testing instances. For the $i$-th training instance $\mathrm{x}_{i}(i \in\{1, \cdots, N\})$ which belongs to the $c$-th $(c \in\{1, \cdots, C\})$ class, $y_{i c}=1$ and $y_{i c^{\prime}}=0\left(c^{\prime} \in\{1, \cdots, C\}\right.$ and $\left.c^{\prime} \neq c\right)$. $Y_{M \times C}^{\prime}$ is unknown and needs to be predicted.

ELM is a single hidden-layer feed-forward neural network (SLFN) and does not require any iterative optimization to input-layer and output-layer weights. ELM determines $Y_{M \times C}^{\prime}$ as follows:

$$
Y_{M \times C}^{\prime}=H_{M \times L}^{\prime} \beta_{L \times C}=H_{M \times L}^{\prime} H_{L \times N}^{\dagger} Y_{N \times C}=\left\{\begin{array}{ll}
H^{\prime}\left(H^{T} H\right)^{-1} H^{T} Y & , \text { if } N ? L \\
H^{\prime} H^{T}\left(H H^{T}\right)^{-1} Y & , \quad \text { if } N<L
\end{array},\right.
$$

where $\beta_{L \times C}$ is the output-layer weight matrix, $L$ is the number of hidden-layer nodes,

$$
H_{N ?}=\left[\begin{array}{c}
h\left(x_{1}\right) \\
M \\
h\left(x_{N}\right)
\end{array}\right]=\left[\begin{array}{ccc}
g\left(w_{1} x_{1}+b_{1}\right) & L & g\left(w_{L} x_{1}+b_{L}\right) \\
M & O & M \\
g\left(w_{1} x_{N}+b_{1}\right) & L & g\left(w_{L} x_{N}+b_{L}\right)
\end{array}\right]
$$

is the hidden-layer output matrix for training data set, $\mathrm{H}_{L \times N}^{\dagger}$ is Moore-Penrose generalized inverse of $H_{N \times L}$,

$$
H_{M ?}^{\prime}=\left[\begin{array}{c}
h\left(x_{1}^{\prime}\right) \\
M \\
h\left(x_{M}^{\prime}\right)
\end{array}\right]=\left[\begin{array}{ccc}
g\left(w_{1} x_{1}^{\prime}+b_{1}\right) & L & g\left(w_{L} x_{1}^{\prime}+b_{L}\right) \\
M & O & M \\
g\left(w_{1} x_{M}^{\prime}+b_{1}\right) & L & g\left(w_{L} x_{M}^{\prime}+b_{L}\right)
\end{array}\right]
$$


is the hidden-layer output matrix for testing data set, $g(v)=\frac{1}{1+e^{-v}}, v \in(-\infty,+\infty)$ is the sigmoid activation function, are the input-layer weight matrix and hidden-layer bias vector which are usually determined by assigning the random numbers obeying Uniform distribution. Our main work in this paper is to use the probability distributions in Fig. 1 to initialize $\mathrm{W}_{D \times L}$ and $\mathrm{b}$ and compare the classification performances of corresponding ELMs.

$$
W_{D \times L}=\left[\begin{array}{lll}
w_{1} & \cdots & w_{L}
\end{array}\right]=\left[\begin{array}{ccc}
w_{11} & \cdots & w_{L 1} \\
\vdots & \ddots & \vdots \\
w_{1 D} & \cdots & w_{L D}
\end{array}\right] \text { and } b=\left[\begin{array}{c}
b_{1} \\
\vdots \\
b_{L}
\end{array}\right]
$$

Table 1. The Details of 30 UCI Data Sets

\begin{tabular}{|c|c|c|c|c|c|}
\hline & Data sets & Attributes & Classes & "Class distribution & Instances \\
\hline 1 & Auto Mpg & 5 & 3 & $245 / 79 / 68$ & 392 \\
\hline 2 & Blood Transfusion & 4 & 2 & $570 / 178$ & 748 \\
\hline 3 & Breast Cancer & 10 & 2 & $458 / 241$ & 699 \\
\hline 4 & Breast CancerW-D & 30 & 2 & $357 / 212$ & 569 \\
\hline 5 & Breast CancerW-P & 33 & 2 & $151 / 47$ & 198 \\
\hline 6 & Cleveland & 13 & 5 & $160 / 54 / 35 / 35 / 13$ & 297 \\
\hline 7 & Credit Approval & 15 & 2 & $383 / 307$ & 690 \\
\hline 8 & Cylinder Bands & 20 & 2 & $312 / 228$ & 540 \\
\hline 9 & Ecoli & 5 & 8 & $143 / 77 / 52 / 35 / 20 / 5 / 2 / 2$ & 336 \\
\hline 10 & Glass Identification & 9 & 7 & $76 / 70 / 29 / 17 / 13 / 9 / 0$ & 214 \\
\hline 11 & Haberman's Survival & 3 & 2 & $225 / 81$ & 306 \\
\hline 12 & Heart Disease & 13 & 2 & $150 / 120$ & 270 \\
\hline 13 & Image Segment & 19 & 7 & $330 \times 7$ & 2310 \\
\hline 14 & Ionosphere & 33 & 2 & $225 / 126$ & 351 \\
\hline 15 & Iris & 4 & 3 & $50 \times 3$ & 150 \\
\hline 16 & Libras Movement & 90 & 15 & $24 \times 15$ & 360 \\
\hline 17 & Magic Telescope & 10 & 2 & $12332 / 6688$ & $19020(10 \%)$ \\
\hline 18 & Musk Version1 & 166 & 2 & $269 / 207$ & 476 \\
\hline 19 & New Thyroid Gland & 5 & 3 & $150 / 35 / 30$ & 215 \\
\hline 20 & Page Blocks & 10 & 5 & $4913 / 329 / 115 / 88 / 28$ & $5473(10 \%)$ \\
\hline 21 & Parkinsons & 22 & 2 & $147 / 48$ & 195 \\
\hline 22 & Pima Indian Diabetes & 8 & 2 & $500 / 268$ & 768 \\
\hline 23 & Sonar & 60 & 2 & $111 / 97$ & 208 \\
\hline 24 & SPECTF Heart & 44 & 2 & $212 / 55$ & 267 \\
\hline 25 & Vehicle Silhouettes & 18 & 4 & $218 / 217 / 212 / 199$ & 846 \\
\hline 26 & Vowel Recognition & 10 & 11 & $48 \times 11$ & 528 \\
\hline 27 & Wine & 13 & 3 & $91 / 59 / 48$ & 178 \\
\hline 28 & Wine Quality-Red & 11 & 6 & $681 / 638 / 199 / 53 / 18 / 10$ & 1599 \\
\hline 29 & WineQuality-White & 11 & 6 & $2198 / 1457 / 880 / 175 / 163 / 20$ & $4898(10 \%)$ \\
\hline 30 & Yeast & 8 & 10 & $463 / 429 / 244 / 163 / 51 / 44 / 35 / 30 / 20 / 5$ & 1484 \\
\hline
\end{tabular}

\section{Impact of Random Initialization on ELM's Generalization Performance}

\subsection{Data Preparation Guideline}

In this experimental study, $30 \mathrm{UCI}$ data sets are used to test the classification performances of ELMs corresponding to different random initializations. The detailed description of 30 data sets is summarized in Table 1. In order to use these data sets more efficiently and specifically, we preprocess them according to the following guidelines:

1) Delete nominal-value attributes. ELMs are mainly used to handle the classification with continuous-value attributes.

2) Replace missing attribute-values. The unsupervised filter named ReplaceMissingValues in Weka [18] is 
used to replace all missing attribute-values in each data set.

3) Reduce large data sets. The unsupervised filter named Resample with the sample-SizePercent 10 in Weka is used to randomly reduce the sizes of three large data sets: Magic Telescope, Page Blocks and Wine Quality-White.

\subsection{Experimental Setup}

Table 2. Training Accuracies of ELMs Corresponding to Uniform Distribution

\begin{tabular}{|c|c|c|c|c|c|c|}
\hline Uniform & $a=0, b=5$ & $a=0, b=10$ & $a=0, b=15$ & $a=0, b=20$ & $a=0, b=25$ & $a=0, b=30$ \\
\hline 1 & $0.864 \pm 0.002$ & $0.803 \pm 0.007$ & $0.727 \pm 0.007$ & $0.681 \pm 0.008$ & $0.654 \pm 0.008$ & $0.639 \pm 0.004$ \\
\hline 2 & $0.811 \pm 0.001$ & $0.808 \pm 0.002$ & $0.794 \pm 0.002$ & $0.783 \pm 0.002$ & $0.777 \pm 0.002$ & $0.771 \pm 0.002$ \\
\hline 3 & $0.974 \pm 0.000$ & $0.939 \pm 0.002$ & $0.890 \pm 0.005$ & $0.852 \pm 0.006$ & $0.803 \pm 0.024$ & $0.721 \pm 0.027$ \\
\hline 4 & $0.698 \pm 0.039$ & $0.627 \pm 0.000$ & $0.627 \pm 0.000$ & $0.627 \pm 0.000$ & $0.627 \pm 0.000$ & $0.627 \pm 0.000$ \\
\hline 5 & $0.763 \pm 0.000$ & $0.763 \pm 0.000$ & $0.763 \pm 0.000$ & $0.763 \pm 0.000$ & $0.763 \pm 0.000$ & $0.763 \pm 0.000$ \\
\hline 6 & $0.670 \pm 0.005$ & $0.589 \pm 0.004$ & $0.556 \pm 0.002$ & $0.546 \pm 0.002$ & $0.541 \pm 0.001$ & $0.540 \pm 0.001$ \\
\hline 7 & $0.814 \pm 0.002$ & $0.807 \pm 0.003$ & $0.774 \pm 0.004$ & $0.742 \pm 0.007$ & $0.719 \pm 0.008$ & $0.697 \pm 0.006$ \\
\hline 8 & $0.579 \pm 0.001$ & $0.578 \pm 0.000$ & $0.578 \pm 0.000$ & $0.578 \pm 0.000$ & $0.578 \pm 0.000$ & $0.578 \pm 0.000$ \\
\hline 9 & $0.880 \pm 0.003$ & $0.682 \pm 0.013$ & $0.541 \pm 0.024$ & $0.440 \pm 0.014$ & $0.429 \pm 0.007$ & $0.426 \pm 0.003$ \\
\hline 10 & $0.800 \pm 0.005$ & $0.616 \pm 0.015$ & $0.402 \pm 0.014$ & $0.363 \pm 0.005$ & $0.358 \pm 0.003$ & $0.357 \pm 0.002$ \\
\hline 11 & $0.798 \pm 0.003$ & $0.782 \pm 0.003$ & $0.770 \pm 0.003$ & $0.759 \pm 0.003$ & $0.751 \pm 0.003$ & $0.746 \pm 0.002$ \\
\hline 12 & $0.770 \pm 0.006$ & $0.607 \pm 0.022$ & $0.555 \pm 0.001$ & $0.555 \pm 0.000$ & $0.556 \pm 0.000$ & $0.556 \pm 0.000$ \\
\hline 13 & $0.797 \pm 0.011$ & $0.298 \pm 0.014$ & $0.169 \pm 0.010$ & $0.146 \pm 0.004$ & $0.144 \pm 0.003$ & $0.143 \pm 0.003$ \\
\hline 14 & $0.641 \pm 0.000$ & $0.641 \pm 0.000$ & $0.641 \pm 0.000$ & $0.641 \pm 0.000$ & $0.641 \pm 0.000$ & $0.641 \pm 0.000$ \\
\hline 15 & $0.985 \pm 0.002$ & $0.916 \pm 0.009$ & $0.804 \pm 0.011$ & $0.720 \pm 0.016$ & $0.647 \pm 0.019$ & $0.579 \pm 0.021$ \\
\hline 16 & $0.068 \pm 0.000$ & $0.068 \pm 0.000$ & $0.068 \pm 0.000$ & $0.068 \pm 0.000$ & $0.068 \pm 0.000$ & $0.068 \pm 0.000$ \\
\hline 17 & $0.825 \pm 0.003$ & $0.703 \pm 0.017$ & $0.643 \pm 0.005$ & $0.641 \pm 0.000$ & $0.641 \pm 0.000$ & $0.641 \pm 0.000$ \\
\hline 18 & $0.565 \pm 0.000$ & $0.565 \pm 0.000$ & $0.565 \pm 0.000$ & $0.565 \pm 0.000$ & $0.565 \pm 0.000$ & $0.565 \pm 0.000$ \\
\hline 19 & $0.954 \pm 0.001$ & $0.946 \pm 0.001$ & $0.933 \pm 0.002$ & $0.923 \pm 0.002$ & $0.918 \pm 0.002$ & $0.916 \pm 0.001$ \\
\hline 20 & $0.967 \pm 0.001$ & $0.923 \pm 0.004$ & $0.904 \pm 0.002$ & $0.898 \pm 0.001$ & $0.895 \pm 0.001$ & $0.892 \pm 0.001$ \\
\hline 21 & $0.873 \pm 0.007$ & $0.756 \pm 0.002$ & $0.754 \pm 0.000$ & $0.754 \pm 0.000$ & $0.754 \pm 0.000$ & $0.754 \pm 0.000$ \\
\hline 22 & $0.772 \pm 0.003$ & $0.677 \pm 0.007$ & $0.656 \pm 0.001$ & $0.652 \pm 0.001$ & $0.651 \pm 0.000$ & $0.651 \pm 0.000$ \\
\hline 23 & $0.534 \pm 0.000$ & $0.534 \pm 0.000$ & $0.534 \pm 0.000$ & $0.534 \pm 0.000$ & $0.534 \pm 0.000$ & $0.534 \pm 0.000$ \\
\hline 24 & $0.794 \pm 0.000$ & $0.794 \pm 0.000$ & $0.794 \pm 0.000$ & $0.794 \pm 0.000$ & $0.794 \pm 0.000$ & $0.794 \pm 0.000$ \\
\hline 25 & $0.676 \pm 0.007$ & $0.450 \pm 0.008$ & $0.360 \pm 0.010$ & $0.298 \pm 0.009$ & $0.275 \pm 0.005$ & $0.267 \pm 0.003$ \\
\hline 26 & $0.582 \pm 0.014$ & $0.107 \pm 0.008$ & $0.093 \pm 0.001$ & $0.093 \pm 0.001$ & $0.093 \pm 0.001$ & $0.093 \pm 0.000$ \\
\hline 27 & $0.825 \pm 0.009$ & $0.424 \pm 0.018$ & $0.399 \pm 0.000$ & $0.399 \pm 0.000$ & $0.399 \pm 0.000$ & $0.399 \pm 0.000$ \\
\hline 28 & $0.614 \pm 0.003$ & $0.518 \pm 0.008$ & $0.438 \pm 0.006$ & $0.426 \pm 0.001$ & $0.426 \pm 0.000$ & $0.426 \pm 0.000$ \\
\hline 29 & $0.629 \pm 0.005$ & $0.488 \pm 0.006$ & $0.450 \pm 0.002$ & $0.446 \pm 0.001$ & $0.446 \pm 0.000$ & $0.446 \pm 0.000$ \\
\hline 30 & $0.602 \pm 0.002$ & $0.485 \pm 0.012$ & $0.352 \pm 0.010$ & $0.323 \pm 0.004$ & $0.315 \pm 0.002$ & $0.313 \pm 0.001$ \\
\hline
\end{tabular}

$\square \mathrm{a}=0, \mathrm{~b}=5 \quad \square \mathrm{a}=0, \mathrm{~b}=10 \square \mathrm{a}=0, \mathrm{~b}=15 \square \mathrm{a}=0, \mathrm{~b}=20 \quad \boldsymbol{Q}_{\mathrm{a}}=0, \mathrm{~b}=25 \quad \square \mathrm{a}=0, \mathrm{~b}=30$

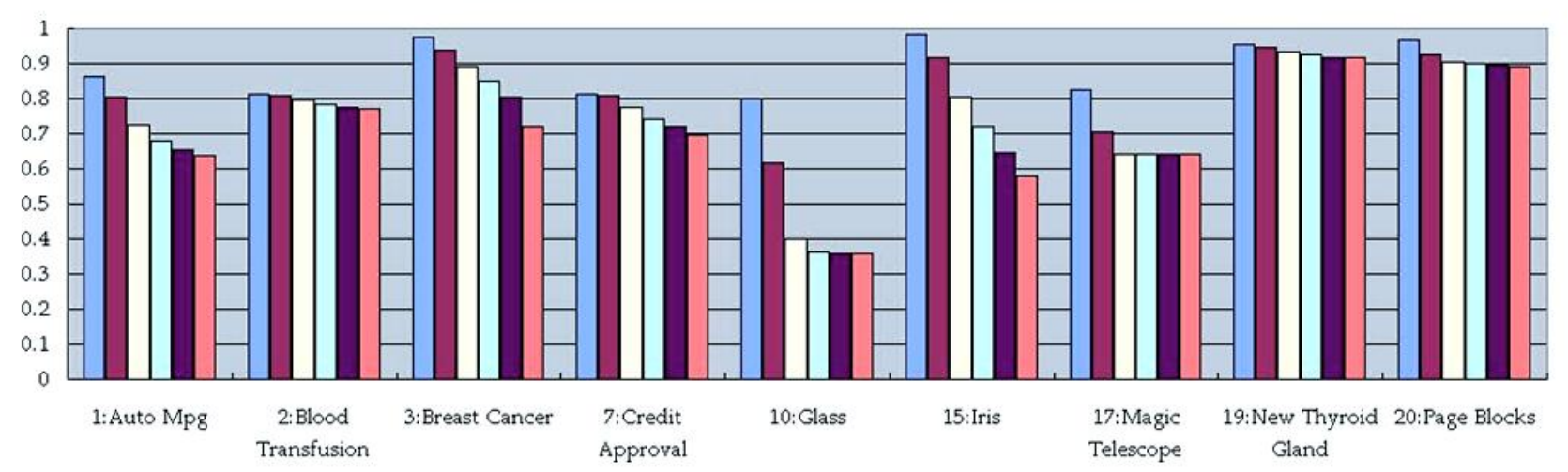

Fig. 2. Training accuracies of 6 ELMs corresponding to 6 Uniform distributions on 9 representative data sets.

The parameter pairs $(a, b),(k, \theta)$ and $(\mu, \sigma)$ in Uniform, Gamma, and Normal distributions are respectively initialized as 6 different two-tuples: $(0,5),(0,10),(0,15),(0,20),(0,25)$ and $(0,30)$ for Uniform; $(9,0.1),(9$, 
0.3), (9, 0.5), (9, 0.7), (9, 0.9) and $(9,1.1)$ for Gamma; and(0, 0.05), $(0,0.1),(0,0.15),(0,0.2),(0,0.25)$ and $(0$, 0.3) for Normal. For each distribution, we use 10-fold cross-validation to get the training and testing accuracies corresponding to 6 ELMs (i.e., initializing the input-layer weights and hidden-layer biases with random numbers corresponding to 6 different variances respectively) on every data set. In order to make the experimental results more reliable, 10-fold cross-validation on each data set is repeated 100 times for each ELM and the average values of 100 training and testing accuracies are used as finally experimental results. In every time of 10 -fold cross-validation, the different ELMs are trained on the same training sets and evaluated on same testing set and the random initializations to input-layer weights and hidden-layer biases are independent.

Table 3. Testing Accuracies of ELMs Corresponding to Uniform Distribution

\begin{tabular}{|c|c|c|c|c|c|c|}
\hline Uniform & $a=0, b=5$ & $a=0, b=10$ & $a=0, b=15$ & $a=0, b=20$ & $a=0, b=25$ & $a=0, b=30$ \\
\hline 1 & $0.804 \pm 0.012$ & $0.759 \pm 0.012$ & $0.704 \pm 0.012$ & $0.671 \pm 0.012$ & $0.646 \pm 0.011$ & $0.634 \pm 0.006$ \\
\hline 2 & $0.797 \pm 0.005$ & $0.796 \pm 0.004$ & $0.785 \pm 0.005$ & $0.774 \pm 0.006$ & $0.772 \pm 0.005$ & $0.764 \pm 0.005$ \\
\hline 3 & $0.965 \pm 0.003$ & $0.929 \pm 0.006$ & $0.882 \pm 0.008$ & $0.845 \pm 0.010$ & $0.797 \pm 0.023$ & $0.715 \pm 0.030$ \\
\hline 4 & $0.684 \pm 0.039$ & $0.627 \pm 0.000$ & $0.627 \pm 0.000$ & $0.627 \pm 0.000$ & $0.627 \pm 0.000$ & $0.627 \pm 0.000$ \\
\hline 5 & $0.761 \pm 0.003$ & $0.763 \pm 0.000$ & $0.763 \pm 0.000$ & $0.763 \pm 0.000$ & $0.763 \pm 0.000$ & $0.763 \pm 0.000$ \\
\hline 6 & $0.529 \pm 0.014$ & $0.526 \pm 0.010$ & $0.528 \pm 0.007$ & $0.532 \pm 0.006$ & $0.536 \pm 0.003$ & $0.538 \pm 0.003$ \\
\hline 7 & $0.775 \pm 0.008$ & $0.774 \pm 0.009$ & $0.748 \pm 0.008$ & $0.724 \pm 0.011$ & $0.704 \pm 0.013$ & $0.684 \pm 0.015$ \\
\hline 8 & $0.579 \pm 0.002$ & $0.578 \pm 0.000$ & $0.578 \pm 0.000$ & $0.578 \pm 0.000$ & $0.578 \pm 0.000$ & $0.578 \pm 0.000$ \\
\hline 9 & $0.830 \pm 0.013$ & $0.655 \pm 0.014$ & $0.530 \pm 0.022$ & $0.437 \pm 0.013$ & $0.428 \pm 0.006$ & $0.426 \pm 0.002$ \\
\hline 10 & $0.659 \pm 0.019$ & $0.569 \pm 0.029$ & $0.381 \pm 0.026$ & $0.359 \pm 0.011$ & $0.357 \pm 0.004$ & $0.355 \pm 0.003$ \\
\hline 11 & $0.715 \pm 0.011$ & $0.730 \pm 0.012$ & $0.739 \pm 0.011$ & $0.734 \pm 0.011$ & $0.733 \pm 0.008$ & $0.731 \pm 0.008$ \\
\hline 12 & $0.698 \pm 0.022$ & $0.589 \pm 0.025$ & $0.553 \pm 0.003$ & $0.555 \pm 0.001$ & $0.556 \pm 0.000$ & $0.556 \pm 0.000$ \\
\hline 13 & $0.791 \pm 0.013$ & $0.298 \pm 0.015$ & $0.169 \pm 0.010$ & $0.146 \pm 0.004$ & $0.144 \pm 0.003$ & $0.142 \pm 0.003$ \\
\hline 14 & $0.641 \pm 0.000$ & $0.641 \pm 0.000$ & $0.641 \pm 0.000$ & $0.641 \pm 0.000$ & $0.641 \pm 0.000$ & $0.641 \pm 0.000$ \\
\hline 15 & $0.943 \pm 0.009$ & $0.867 \pm 0.022$ & $0.773 \pm 0.020$ & $0.698 \pm 0.021$ & $0.622 \pm 0.028$ & $0.563 \pm 0.028$ \\
\hline 16 & $0.056 \pm 0.000$ & $0.056 \pm 0.000$ & $0.056 \pm 0.000$ & $0.056 \pm 0.000$ & $0.056 \pm 0.000$ & $0.056 \pm 0.000$ \\
\hline 17 & $0.819 \pm 0.004$ & $0.701 \pm 0.017$ & $0.643 \pm 0.004$ & $0.641 \pm 0.000$ & $0.641 \pm 0.000$ & $0.641 \pm 0.000$ \\
\hline 18 & $0.565 \pm 0.000$ & $0.565 \pm 0.000$ & $0.565 \pm 0.000$ & $0.565 \pm 0.000$ & $0.565 \pm 0.000$ & $0.565 \pm 0.000$ \\
\hline 19 & $0.940 \pm 0.003$ & $0.937 \pm 0.003$ & $0.927 \pm 0.003$ & $0.920 \pm 0.004$ & $0.916 \pm 0.003$ & $0.914 \pm 0.002$ \\
\hline 20 & $0.939 \pm 0.005$ & $0.900 \pm 0.006$ & $0.890 \pm 0.005$ & $0.889 \pm 0.003$ & $0.889 \pm 0.002$ & $0.888 \pm 0.002$ \\
\hline 21 & $0.822 \pm 0.017$ & $0.754 \pm 0.005$ & $0.754 \pm 0.000$ & $0.754 \pm 0.000$ & $0.754 \pm 0.000$ & $0.754 \pm 0.000$ \\
\hline 22 & $0.744 \pm 0.010$ & $0.661 \pm 0.010$ & $0.648 \pm 0.004$ & $0.648 \pm 0.003$ & $0.649 \pm 0.002$ & $0.650 \pm 0.001$ \\
\hline 23 & $0.534 \pm 0.000$ & $0.534 \pm 0.000$ & $0.534 \pm 0.000$ & $0.534 \pm 0.000$ & $0.534 \pm 0.000$ & $0.534 \pm 0.000$ \\
\hline 24 & $0.794 \pm 0.000$ & $0.794 \pm 0.000$ & $0.794 \pm 0.000$ & $0.794 \pm 0.000$ & $0.794 \pm 0.000$ & $0.794 \pm 0.000$ \\
\hline 25 & $0.616 \pm 0.014$ & $0.423 \pm 0.011$ & $0.345 \pm 0.012$ & $0.289 \pm 0.012$ & $0.272 \pm 0.007$ & $0.265 \pm 0.004$ \\
\hline 26 & $0.521 \pm 0.022$ & $0.092 \pm 0.011$ & $0.076 \pm 0.002$ & $0.076 \pm 0.002$ & $0.076 \pm 0.001$ & $0.076 \pm 0.000$ \\
\hline 27 & $0.758 \pm 0.022$ & $0.420 \pm 0.022$ & $0.399 \pm 0.000$ & $0.399 \pm 0.000$ & $0.399 \pm 0.000$ & $0.399 \pm 0.000$ \\
\hline 28 & $0.589 \pm 0.006$ & $0.512 \pm 0.013$ & $0.436 \pm 0.009$ & $0.426 \pm 0.002$ & $0.426 \pm 0.000$ & $0.426 \pm 0.000$ \\
\hline 29 & $0.526 \pm 0.015$ & $0.457 \pm 0.011$ & $0.444 \pm 0.005$ & $0.446 \pm 0.002$ & $0.446 \pm 0.001$ & $0.446 \pm 0.000$ \\
\hline 30 & $0.579 \pm 0.004$ & $0.478 \pm 0.014$ & $0.349 \pm 0.009$ & $0.322 \pm 0.006$ & $0.314 \pm 0.002$ & $0.313 \pm 0.002$ \\
\hline
\end{tabular}

\subsection{Experimental Result and Analysis}

The training and testing accuracies of 18 ELMs (The numbers of hidden-layer nodes are 50) corresponding to 3 different kinds of probability distributions on $30 \mathrm{UCI}$ data sets are summarized in Tables 2-3, 4-5, and 6-7, respectively. For each distribution, we use Wilcoxon signed ranks test and Friedman test [17] to conduct the statistical analysis to the above-mentioned experimental results. The former is to assess whether the classification accuracies of two ELMs differ on a single data set, while the latter is to test whether or not the classification accuracies of 6 ELMs are equal on all data sets. For a given distribution, we only give the detailed procedures of these two statistical tests based on training accuracies of 6 ELMs. The testing accuracies can be handled in the same way. The compared objects of Wilcoxon signed-ranks test are the respective 100 training accuracies of two ELMs (ELM 1 and ELM $)$ on a single data set. 


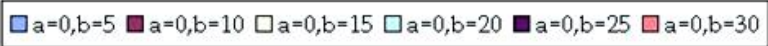

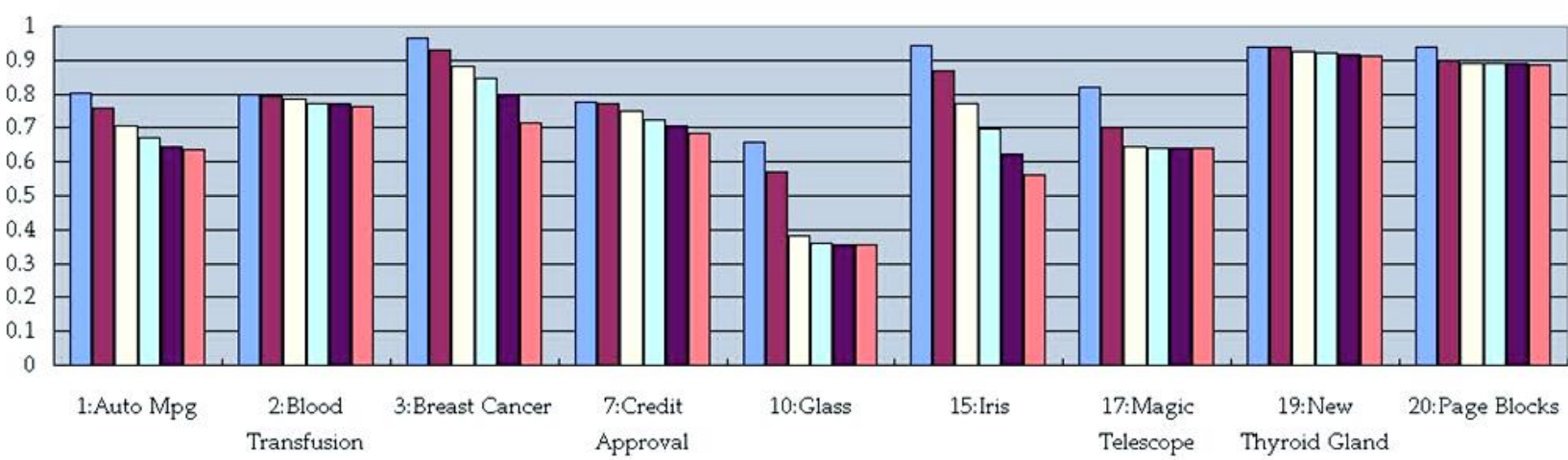

Fig. 3. Testing accuracies of 6 ELMs corresponding to 6 Uniform distributions on 9 representative data sets.

Table 4. Training Accuracies of ELMs Corresponding to Gamma Distribution

\begin{tabular}{|c|c|c|c|c|c|c|}
\hline Gamma & $k=9, \theta=0.1$ & $k=9, \theta=0.3$ & $k=9, \theta=0.5$ & $k=9, \theta=0.7$ & $k=9, \theta=0.9$ & $k=9, \theta=1.1$ \\
\hline 1 & $0.863 \pm 0.002$ & $0.851 \pm 0.003$ & $0.789 \pm 0.006$ & $0.705 \pm 0.006$ & $0.647 \pm 0.004$ & $0.628 \pm 0.002$ \\
\hline 2 & $0.806 \pm 0.001$ & $0.809 \pm 0.001$ & $0.804 \pm 0.002$ & $0.791 \pm 0.002$ & $0.779 \pm 0.002$ & $0.773 \pm 0.002$ \\
\hline 3 & $0.979 \pm 0.001$ & $0.972 \pm 0.000$ & $0.941 \pm 0.002$ & $0.900 \pm 0.002$ & $0.855 \pm 0.004$ & $0.830 \pm 0.020$ \\
\hline 4 & $0.935 \pm 0.002$ & $0.626 \pm 0.000$ & $0.627 \pm 0.000$ & $0.627 \pm 0.000$ & $0.627 \pm 0.000$ & $0.627 \pm 0.000$ \\
\hline 5 & $0.817 \pm 0.003$ & $0.763 \pm 0.000$ & $0.763 \pm 0.000$ & $0.763 \pm 0.000$ & $0.763 \pm 0.000$ & $0.763 \pm 0.000$ \\
\hline 6 & $0.698 \pm 0.004$ & $0.652 \pm 0.004$ & $0.581 \pm 0.003$ & $0.546 \pm 0.002$ & $0.539 \pm 0.001$ & $0.539 \pm 0.000$ \\
\hline 7 & $0.805 \pm 0.002$ & $0.816 \pm 0.002$ & $0.809 \pm 0.002$ & $0.781 \pm 0.003$ & $0.744 \pm 0.004$ & $0.711 \pm 0.004$ \\
\hline 8 & $0.688 \pm 0.005$ & $0.578 \pm 0.000$ & $0.578 \pm 0.000$ & $0.578 \pm 0.000$ & $0.578 \pm 0.000$ & $0.578 \pm 0.000$ \\
\hline 9 & $0.899 \pm 0.002$ & $0.851 \pm 0.004$ & $0.639 \pm 0.005$ & $0.483 \pm 0.029$ & $0.426 \pm 0.000$ & $0.426 \pm 0.000$ \\
\hline 10 & $0.819 \pm 0.005$ & $0.785 \pm 0.005$ & $0.521 \pm 0.021$ & $0.355 \pm 0.002$ & $0.355 \pm 0.000$ & $0.355 \pm 0.000$ \\
\hline 11 & $0.782 \pm 0.002$ & $0.785 \pm 0.002$ & $0.778 \pm 0.003$ & $0.762 \pm 0.002$ & $0.750 \pm 0.002$ & $0.743 \pm 0.001$ \\
\hline 12 & $0.857 \pm 0.004$ & $0.738 \pm 0.004$ & $0.554 \pm 0.001$ & $0.556 \pm 0.000$ & $0.556 \pm 0.000$ & $0.556 \pm 0.000$ \\
\hline 13 & $0.960 \pm 0.002$ & $0.667 \pm 0.018$ & $0.232 \pm 0.015$ & $0.178 \pm 0.000$ & $0.178 \pm 0.000$ & $0.178 \pm 0.000$ \\
\hline 14 & $0.693 \pm 0.003$ & $0.641 \pm 0.000$ & $0.641 \pm 0.000$ & $0.641 \pm 0.000$ & $0.641 \pm 0.000$ & $0.641 \pm 0.000$ \\
\hline 15 & $0.985 \pm 0.001$ & $0.979 \pm 0.002$ & $0.890 \pm 0.008$ & $0.785 \pm 0.008$ & $0.671 \pm 0.011$ & $0.563 \pm 0.013$ \\
\hline 16 & $0.068 \pm 0.002$ & $0.068 \pm 0.000$ & $0.068 \pm 0.000$ & $0.068 \pm 0.000$ & $0.068 \pm 0.000$ & $0.068 \pm 0.000$ \\
\hline 17 & $0.851 \pm 0.001$ & $0.807 \pm 0.002$ & $0.646 \pm 0.005$ & $0.641 \pm 0.000$ & $0.641 \pm 0.000$ & $0.641 \pm 0.000$ \\
\hline 18 & $0.565 \pm 0.000$ & $0.565 \pm 0.000$ & $0.565 \pm 0.000$ & $0.565 \pm 0.000$ & $0.565 \pm 0.000$ & $0.565 \pm 0.000$ \\
\hline 19 & $0.953 \pm 0.001$ & $0.951 \pm 0.001$ & $0.946 \pm 0.001$ & $0.930 \pm 0.001$ & $0.917 \pm 0.001$ & $0.914 \pm 0.000$ \\
\hline 20 & $0.969 \pm 0.001$ & $0.970 \pm 0.001$ & $0.911 \pm 0.002$ & $0.901 \pm 0.001$ & $0.897 \pm 0.001$ & $0.892 \pm 0.001$ \\
\hline 21 & $0.981 \pm 0.003$ & $0.790 \pm 0.005$ & $0.754 \pm 0.000$ & $0.754 \pm 0.000$ & $0.754 \pm 0.000$ & $0.754 \pm 0.000$ \\
\hline 22 & $0.799 \pm 0.001$ & $0.763 \pm 0.002$ & $0.666 \pm 0.006$ & $0.652 \pm 0.001$ & $0.651 \pm 0.000$ & $0.651 \pm 0.000$ \\
\hline 23 & $0.571 \pm 0.005$ & $0.534 \pm 0.000$ & $0.534 \pm 0.000$ & $0.534 \pm 0.000$ & $0.534 \pm 0.000$ & $0.534 \pm 0.000$ \\
\hline 24 & $0.794 \pm 0.000$ & $0.794 \pm 0.000$ & $0.794 \pm 0.000$ & $0.794 \pm 0.000$ & $0.794 \pm 0.000$ & $0.794 \pm 0.000$ \\
\hline 25 & $0.780 \pm 0.003$ & $0.642 \pm 0.006$ & $0.437 \pm 0.005$ & $0.341 \pm 0.010$ & $0.267 \pm 0.002$ & $0.259 \pm 0.001$ \\
\hline 26 & $0.858 \pm 0.004$ & $0.357 \pm 0.014$ & $0.093 \pm 0.000$ & $0.093 \pm 0.000$ & $0.092 \pm 0.001$ & $0.093 \pm 0.000$ \\
\hline 27 & $0.988 \pm 0.001$ & $0.728 \pm 0.007$ & $0.399 \pm 0.000$ & $0.399 \pm 0.000$ & $0.399 \pm 0.000$ & $0.399 \pm 0.000$ \\
\hline 28 & $0.629 \pm 0.002$ & $0.606 \pm 0.002$ & $0.504 \pm 0.007$ & $0.426 \pm 0.001$ & $0.426 \pm 0.000$ & $0.426 \pm 0.000$ \\
\hline 29 & $0.652 \pm 0.004$ & $0.613 \pm 0.004$ & $0.513 \pm 0.002$ & $0.490 \pm 0.002$ & $0.486 \pm 0.000$ & $0.486 \pm 0.000$ \\
\hline 30 & $0.622 \pm 0.001$ & $0.585 \pm 0.002$ & $0.454 \pm 0.008$ & $0.325 \pm 0.002$ & $0.313 \pm 0.001$ & $0.312 \pm 0.000$ \\
\hline
\end{tabular}

1) $a_{i}^{(1)}$ and $a_{i}^{(2)}$ are the training accuracies of $\operatorname{ELM}_{1}$ and $\operatorname{ELM}_{2}$ in the $i$-th $(i \in\{1, \cdots, 100\})$ run of 10 -fold cross-validation, respectively.

2) $d_{i}=a_{i}^{(1)}-a_{i}^{(2)}$ is the difference between the training accuracies of ELM ${ }_{1}$ and $\mathrm{ELM}_{2}$ in the $i$-th run of 10 -fold cross-validation.

3) Increasingly order $d_{i}(i=1, \cdots, 100)$ starting with the smallest as 1 . For $d_{i}$ with same absolute values, the average of their ranks is assigned as the new rank. Let $\operatorname{rank}\left(d_{i}\right)$ denote the rank of $d_{i}$.

4) Let $R_{+}$and $R_{-}$denote the sums of ranks for the runs on which ELM 1 is better and worse than ELM 2 
respectively. $R_{+}$and $R_{-}$can be calculated as follows:

$$
R_{+}=\sum_{\substack{i=1 \\ d_{i}>0}}^{100} \operatorname{rank}\left(d_{i}\right)+\frac{1}{2} \sum_{\substack{i=1 \\ d_{i}=0}}^{100} \operatorname{rank}\left(d_{i}\right)
$$

and

5) Construct the statistic $z=\frac{\min \left(R_{+}, R_{-}\right)-\frac{1}{4} m(m+1)}{\sqrt{\frac{1}{24} m(m+1)(2 m+1)}}$ based on the null-hypothesis which indicates that $E_{L} M_{1}$ and $E_{L} M_{2}$ have the equal training accuracy on a given data set, $m$ is the times of 10 -fold cross-validations. In our study, $m=100$. When $m \geq 30$, the distribution of $z$ is approximately normal.

6) Set the significance level $\alpha=0: 1$. If $z_{>} z_{\alpha / 2}$ or $Z<Z_{-\alpha / 2}$, the null-hypothesis can be rejected, i.e., there are some differences between ELM1 and ELM2; else, we accept the null-hypothesis. The value $z_{0.1 / 2}=z_{0.05}=$ 1.645 can be found from any statistical textbook.

$$
R_{-}=\sum_{\substack{i=1 \\ d_{i}<0}}^{100} \operatorname{rank}\left(d_{i}\right)+\frac{1}{2} \sum_{\substack{i=1 \\ d_{i}=0}}^{100} \operatorname{rank}\left(d_{i}\right)
$$

\section{$\square K=9, \theta=0.1 \square K=9, \theta=0.3 \square K=9, \theta=0.5 \square K=9, \theta=0.7 \square K=9, \theta=0.9 \square K=9, \theta=1.1$}

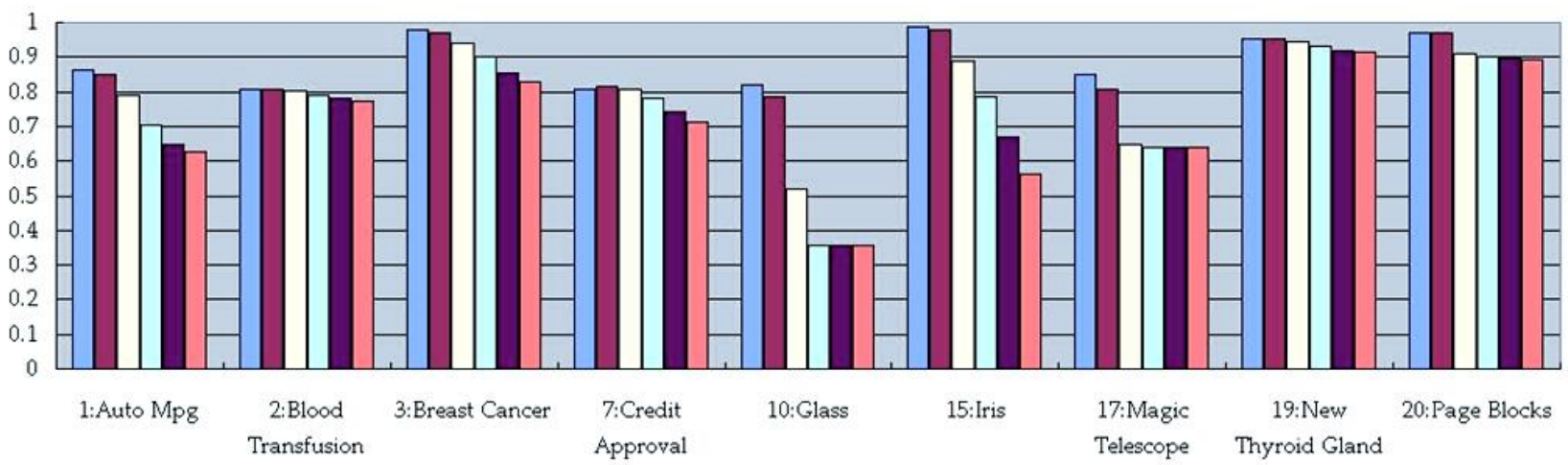

Fig. 4. Training accuracies of 6 ELMs corresponding to 6 Gamma distributions on 9 representative data sets

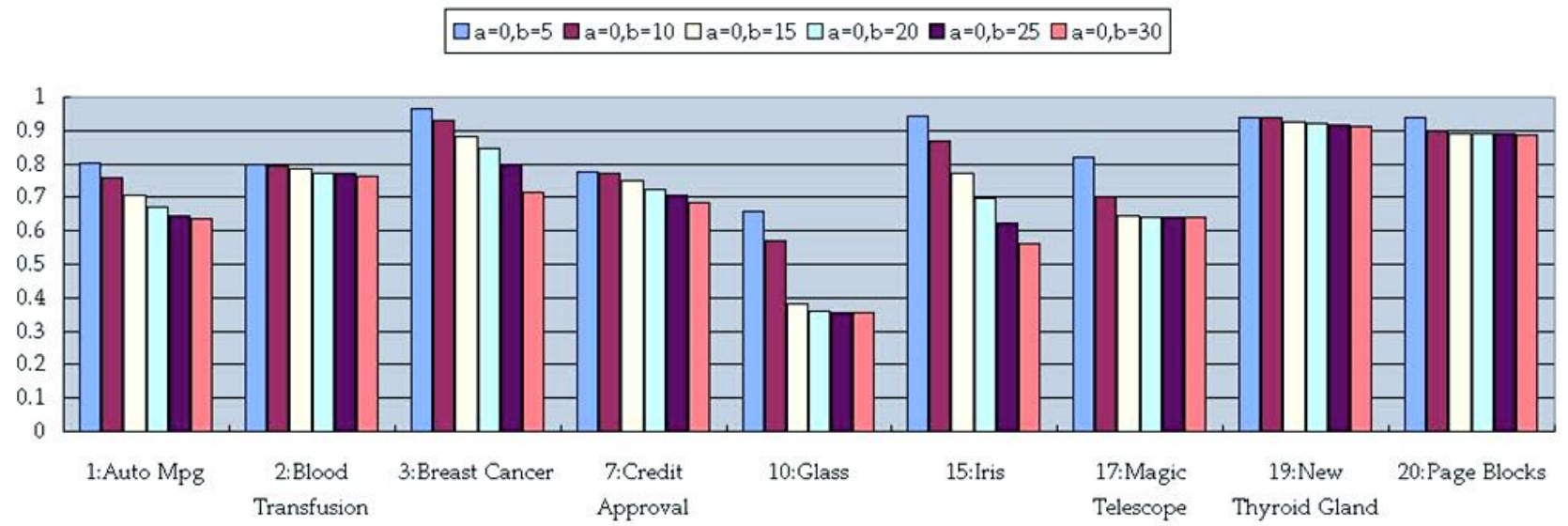

Fig. 5. Testing accuracies of 6 ELMs corresponding to 6 Gamma distributions on 9 representative data sets. 
Table 5. Testing Accuracies of ELMs Corresponding to Gamma Distribution

\begin{tabular}{|c|c|c|c|c|c|c|}
\hline Gamma & $k=9, \theta=0.1$ & $k=9, \theta=0.3$ & $k=9, \theta=0.5$ & $k=9, \theta=0.7$ & $k=9, \theta=0.9$ & $k=9, \theta=1.1$ \\
\hline 1 & $0.806 \pm 0.009$ & $0.798 \pm 0.011$ & $0.754 \pm 0.011$ & $0.688 \pm 0.013$ & $0.640 \pm 0.008$ & $0.627 \pm 0.002$ \\
\hline 2 & $0.795 \pm 0.004$ & $0.797 \pm 0.005$ & $0.794 \pm 0.005$ & $0.783 \pm 0.005$ & $0.774 \pm 0.005$ & $0.770 \pm 0.004$ \\
\hline 3 & $0.965 \pm 0.003$ & $0.965 \pm 0.003$ & $0.929 \pm 0.006$ & $0.892 \pm 0.005$ & $0.849 \pm 0.008$ & $0.825 \pm 0.022$ \\
\hline 4 & $0.916 \pm 0.007$ & $0.623 \pm 0.003$ & $0.627 \pm 0.000$ & $0.627 \pm 0.000$ & $0.627 \pm 0.000$ & $0.627 \pm 0.000$ \\
\hline 5 & $0.707 \pm 0.018$ & $0.763 \pm 0.000$ & $0.763 \pm 0.000$ & $0.763 \pm 0.000$ & $0.763 \pm 0.000$ & $0.763 \pm 0.000$ \\
\hline 6 & $0.548 \pm 0.010$ & $0.532 \pm 0.013$ & $0.530 \pm 0.008$ & $0.532 \pm 0.005$ & $0.537 \pm 0.002$ & $0.539 \pm 0.000$ \\
\hline 7 & $0.770 \pm 0.007$ & $0.779 \pm 0.008$ & $0.775 \pm 0.007$ & $0.755 \pm 0.010$ & $0.724 \pm 0.008$ & $0.699 \pm 0.010$ \\
\hline 8 & $0.616 \pm 0.013$ & $0.578 \pm 0.000$ & $0.578 \pm 0.000$ & $0.578 \pm 0.000$ & $0.578 \pm 0.000$ & $0.578 \pm 0.000$ \\
\hline 9 & $0.854 \pm 0.008$ & $0.809 \pm 0.010$ & $0.620 \pm 0.010$ & $0.480 \pm 0.031$ & $0.426 \pm 0.000$ & $0.426 \pm 0.000$ \\
\hline 10 & $0.648 \pm 0.021$ & $0.679 \pm 0.014$ & $0.488 \pm 0.031$ & $0.355 \pm 0.005$ & $0.355 \pm 0.000$ & $0.355 \pm 0.000$ \\
\hline 11 & $0.735 \pm 0.008$ & $0.732 \pm 0.010$ & $0.738 \pm 0.011$ & $0.738 \pm 0.010$ & $0.736 \pm 0.006$ & $0.732 \pm 0.006$ \\
\hline 12 & $0.775 \pm 0.014$ & $0.691 \pm 0.016$ & $0.549 \pm 0.006$ & $0.556 \pm 0.000$ & $0.556 \pm 0.000$ & $0.556 \pm 0.000$ \\
\hline 13 & $0.924 \pm 0.008$ & $0.650 \pm 0.022$ & $0.231 \pm 0.015$ & $0.178 \pm 0.000$ & $0.178 \pm 0.000$ & $0.178 \pm 0.000$ \\
\hline 14 & $0.686 \pm 0.007$ & $0.641 \pm 0.000$ & $0.641 \pm 0.000$ & $0.641 \pm 0.000$ & $0.641 \pm 0.000$ & $0.641 \pm 0.000$ \\
\hline 15 & $0.956 \pm 0.009$ & $0.946 \pm 0.010$ & $0.856 \pm 0.018$ & $0.765 \pm 0.015$ & $0.655 \pm 0.021$ & $0.548 \pm 0.015$ \\
\hline 16 & $0.056 \pm 0.005$ & $0.056 \pm 0.000$ & $0.056 \pm 0.000$ & $0.056 \pm 0.000$ & $0.056 \pm 0.000$ & $0.056 \pm 0.000$ \\
\hline 17 & $0.842 \pm 0.003$ & $0.803 \pm 0.005$ & $0.646 \pm 0.005$ & $0.641 \pm 0.000$ & $0.641 \pm 0.000$ & $0.641 \pm 0.000$ \\
\hline 18 & $0.565 \pm 0.000$ & $0.565 \pm 0.000$ & $0.565 \pm 0.000$ & $0.565 \pm 0.000$ & $0.565 \pm 0.000$ & $0.565 \pm 0.000$ \\
\hline 19 & $0.941 \pm 0.002$ & $0.941 \pm 0.003$ & $0.939 \pm 0.003$ & $0.926 \pm 0.002$ & $0.915 \pm 0.002$ & $0.913 \pm 0.001$ \\
\hline 20 & $0.939 \pm 0.004$ & $0.941 \pm 0.005$ & $0.892 \pm 0.005$ & $0.889 \pm 0.003$ & $0.887 \pm 0.003$ & $0.884 \pm 0.002$ \\
\hline 21 & $0.894 \pm 0.019$ & $0.766 \pm 0.013$ & $0.754 \pm 0.001$ & $0.754 \pm 0.000$ & $0.754 \pm 0.000$ & $0.754 \pm 0.000$ \\
\hline 22 & $0.767 \pm 0.007$ & $0.742 \pm 0.007$ & $0.655 \pm 0.007$ & $0.648 \pm 0.002$ & $0.650 \pm 0.001$ & $0.651 \pm 0.000$ \\
\hline 23 & $0.573 \pm 0.009$ & $0.534 \pm 0.000$ & $0.534 \pm 0.000$ & $0.534 \pm 0.000$ & $0.534 \pm 0.000$ & $0.534 \pm 0.000$ \\
\hline 24 & $0.794 \pm 0.000$ & $0.794 \pm 0.000$ & $0.794 \pm 0.000$ & $0.794 \pm 0.000$ & $0.794 \pm 0.000$ & $0.794 \pm 0.000$ \\
\hline 25 & $0.718 \pm 0.011$ & $0.586 \pm 0.011$ & $0.413 \pm 0.010$ & $0.329 \pm 0.015$ & $0.263 \pm 0.004$ & $0.259 \pm 0.002$ \\
\hline 26 & $0.776 \pm 0.012$ & $0.322 \pm 0.019$ & $0.076 \pm 0.000$ & $0.076 \pm 0.001$ & $0.076 \pm 0.001$ & $0.076 \pm 0.000$ \\
\hline 27 & $0.943 \pm 0.014$ & $0.707 \pm 0.016$ & $0.399 \pm 0.000$ & $0.399 \pm 0.000$ & $0.399 \pm 0.000$ & $0.399 \pm 0.000$ \\
\hline 28 & $0.599 \pm 0.006$ & $0.583 \pm 0.008$ & $0.500 \pm 0.011$ & $0.426 \pm 0.002$ & $0.426 \pm 0.000$ & $0.426 \pm 0.000$ \\
\hline 29 & $0.549 \pm 0.014$ & $0.533 \pm 0.011$ & $0.503 \pm 0.007$ & $0.489 \pm 0.004$ & $0.486 \pm 0.000$ & $0.486 \pm 0.000$ \\
\hline 30 & $0.594 \pm 0.004$ & $0.567 \pm 0.005$ & $0.450 \pm 0.012$ & $0.324 \pm 0.003$ & $0.312 \pm 0.001$ & $0.312 \pm 0.000$ \\
\hline
\end{tabular}

Table 6. Training Accuracies of ELMs Corresponding to Normal Distribution

\begin{tabular}{|c|c|c|c|c|c|c|}
\hline Normal & $\mu=0, \sigma=0.05$ & $\mu=0, \sigma=0.1$ & $\mu=0, \sigma=0.15$ & $\mu=0, \sigma=0.2$ & $\mu=0, \sigma=0.25$ & $\mu=0, \sigma=0.3$ \\
\hline 1 & $0.812 \pm 0.003$ & $0.838 \pm 0.003$ & $0.850 \pm 0.002$ & $0.852 \pm 0.002$ & $0.854 \pm 0.002$ & $0.855 \pm 0.002$ \\
\hline 2 & $0.791 \pm 0.001$ & $0.795 \pm 0.001$ & $0.795 \pm 0.001$ & $0.796 \pm 0.001$ & $0.798 \pm 0.001$ & $0.800 \pm 0.001$ \\
\hline 3 & $0.972 \pm 0.001$ & $0.972 \pm 0.001$ & $0.972 \pm 0.001$ & $0.972 \pm 0.001$ & $0.972 \pm 0.001$ & $0.972 \pm 0.001$ \\
\hline 4 & $0.971 \pm 0.001$ & $0.971 \pm 0.001$ & $0.971 \pm 0.001$ & $0.972 \pm 0.001$ & $0.973 \pm 0.001$ & $0.974 \pm 0.001$ \\
\hline 5 & $0.895 \pm 0.005$ & $0.893 \pm 0.004$ & $0.895 \pm 0.004$ & $0.895 \pm 0.004$ & $0.894 \pm 0.004$ & $0.893 \pm 0.004$ \\
\hline 6 & $0.636 \pm 0.003$ & $0.682 \pm 0.004$ & $0.699 \pm 0.003$ & $0.706 \pm 0.003$ & $0.707 \pm 0.003$ & $0.707 \pm 0.003$ \\
\hline 7 & $0.772 \pm 0.002$ & $0.794 \pm 0.002$ & $0.801 \pm 0.002$ & $0.802 \pm 0.002$ & $0.803 \pm 0.002$ & $0.803 \pm 0.002$ \\
\hline 8 & $0.722 \pm 0.005$ & $0.721 \pm 0.006$ & $0.722 \pm 0.005$ & $0.722 \pm 0.005$ & $0.722 \pm 0.005$ & $0.722 \pm 0.004$ \\
\hline 9 & $0.890 \pm 0.002$ & $0.901 \pm 0.002$ & $0.904 \pm 0.002$ & $0.906 \pm 0.002$ & $0.906 \pm 0.002$ & $0.906 \pm 0.002$ \\
\hline 10 & $0.779 \pm 0.004$ & $0.817 \pm 0.005$ & $0.817 \pm 0.005$ & $0.817 \pm 0.005$ & $0.819 \pm 0.005$ & $0.816 \pm 0.004$ \\
\hline 11 & $0.772 \pm 0.002$ & $0.782 \pm 0.002$ & $0.782 \pm 0.002$ & $0.783 \pm 0.002$ & $0.785 \pm 0.003$ & $0.788 \pm 0.002$ \\
\hline 12 & $0.888 \pm 0.004$ & $0.888 \pm 0.003$ & $0.887 \pm 0.004$ & $0.888 \pm 0.004$ & $0.889 \pm 0.003$ & $0.889 \pm 0.003$ \\
\hline 13 & $0.946 \pm 0.003$ & $0.950 \pm 0.003$ & $0.950 \pm 0.002$ & $0.951 \pm 0.002$ & $0.951 \pm 0.003$ & $0.951 \pm 0.002$ \\
\hline 14 & $0.929 \pm 0.003$ & $0.930 \pm 0.003$ & $0.930 \pm 0.003$ & $0.929 \pm 0.003$ & $0.931 \pm 0.003$ & $0.932 \pm 0.004$ \\
\hline 15 & $0.984 \pm 0.002$ & $0.986 \pm 0.001$ & $0.986 \pm 0.001$ & $0.986 \pm 0.001$ & $0.986 \pm 0.001$ & $0.986 \pm 0.001$ \\
\hline 16 & $0.806 \pm 0.004$ & $0.832 \pm 0.004$ & $0.851 \pm 0.005$ & $0.863 \pm 0.004$ & $0.870 \pm 0.004$ & $0.873 \pm 0.004$ \\
\hline 17 & $0.843 \pm 0.001$ & $0.846 \pm 0.001$ & $0.845 \pm 0.001$ & $0.846 \pm 0.001$ & $0.846 \pm 0.001$ & $0.846 \pm 0.001$ \\
\hline 18 & $0.844 \pm 0.005$ & $0.843 \pm 0.005$ & $0.841 \pm 0.005$ & $0.838 \pm 0.006$ & $0.837 \pm 0.006$ & $0.834 \pm 0.006$ \\
\hline 19 & $0.938 \pm 0.001$ & $0.945 \pm 0.001$ & $0.949 \pm 0.001$ & $0.951 \pm 0.001$ & $0.953 \pm 0.001$ & $0.954 \pm 0.001$ \\
\hline 20 & $0.951 \pm 0.001$ & $0.962 \pm 0.001$ & $0.965 \pm 0.001$ & $0.966 \pm 0.001$ & $0.967 \pm 0.001$ & $0.967 \pm 0.001$ \\
\hline 21 & $0.934 \pm 0.003$ & $0.937 \pm 0.003$ & $0.938 \pm 0.004$ & $0.937 \pm 0.003$ & $0.937 \pm 0.004$ & $0.938 \pm 0.004$ \\
\hline 22 & $0.799 \pm 0.002$ & $0.802 \pm 0.002$ & $0.802 \pm 0.002$ & $0.803 \pm 0.002$ & $0.803 \pm 0.002$ & $0.803 \pm 0.002$ \\
\hline 23 & $0.886 \pm 0.005$ & $0.887 \pm 0.005$ & $0.888 \pm 0.005$ & $0.890 \pm 0.005$ & $0.893 \pm 0.005$ & $0.895 \pm 0.005$ \\
\hline 24 & $0.855 \pm 0.003$ & $0.854 \pm 0.004$ & $0.853 \pm 0.004$ & $0.852 \pm 0.004$ & $0.851 \pm 0.004$ & $0.850 \pm 0.005$ \\
\hline 25 & $0.742 \pm 0.004$ & $0.770 \pm 0.003$ & $0.769 \pm 0.003$ & $0.769 \pm 0.005$ & $0.770 \pm 0.004$ & $0.771 \pm 0.003$ \\
\hline 26 & $0.860 \pm 0.005$ & $0.863 \pm 0.005$ & $0.863 \pm 0.005$ & $0.862 \pm 0.004$ & $0.862 \pm 0.005$ & $0.863 \pm 0.005$ \\
\hline
\end{tabular}




\begin{tabular}{lllllll}
\hline 27 & $1.000 \pm 0.000$ & $1.000 \pm 0.000$ & $1.000 \pm 0.000$ & $1.000 \pm 0.000$ & $1.000 \pm 0.000$ & $1.000 \pm 0.000$ \\
\hline 28 & $0.624 \pm 0.002$ & $0.627 \pm 0.002$ & $0.627 \pm 0.003$ & $0.627 \pm 0.002$ & $0.627 \pm 0.002$ & $0.627 \pm 0.002$ \\
\hline 29 & $0.627 \pm 0.005$ & $0.650 \pm 0.004$ & $0.649 \pm 0.004$ & $0.648 \pm 0.004$ & $0.648 \pm 0.005$ & $0.649 \pm 0.004$ \\
\hline 30 & $0.617 \pm 0.002$ & $0.624 \pm 0.002$ & $0.624 \pm 0.001$ & $0.623 \pm 0.002$ & $0.624 \pm 0.002$ & $0.624 \pm 0.002$ \\
\hline \hline
\end{tabular}

Table 7. Testing Accuracies of ELMs Corresponding to Normal Distribution

\begin{tabular}{|c|c|c|c|c|c|c|}
\hline Normal & $\mu=0, \sigma=0.05$ & $\mu=0, \sigma=0.1$ & $\mu=0, \sigma=0.15$ & $\mu=0, \sigma=0.2$ & $\mu=0, \sigma=0.25$ & $\mu=0, \sigma=0.3$ \\
\hline 1 & $0.781 \pm 0.009$ & $0.800 \pm 0.009$ & $0.798 \pm 0.009$ & $0.797 \pm 0.009$ & $0.798 \pm 0.009$ & $0.799 \pm 0.010$ \\
\hline 2 & $0.786 \pm 0.004$ & $0.788 \pm 0.002$ & $0.788 \pm 0.003$ & $0.787 \pm 0.003$ & $0.788 \pm 0.003$ & $0.788 \pm 0.004$ \\
\hline 3 & $0.962 \pm 0.003$ & $0.963 \pm 0.003$ & $0.963 \pm 0.003$ & $0.963 \pm 0.003$ & $0.963 \pm 0.003$ & $0.963 \pm 0.003$ \\
\hline 4 & $0.955 \pm 0.004$ & $0.955 \pm 0.005$ & $0.955 \pm 0.004$ & $0.955 \pm 0.005$ & $0.957 \pm 0.005$ & $0.958 \pm 0.005$ \\
\hline 5 & $0.783 \pm 0.018$ & $0.779 \pm 0.020$ & $0.771 \pm 0.016$ & $0.775 \pm 0.021$ & $0.774 \pm 0.022$ & $0.777 \pm 0.018$ \\
\hline 6 & $0.557 \pm 0.011$ & $0.550 \pm 0.012$ & $0.554 \pm 0.014$ & $0.552 \pm 0.011$ & $0.552 \pm 0.013$ & $0.550 \pm 0.013$ \\
\hline 7 & $0.753 \pm 0.006$ & $0.762 \pm 0.007$ & $0.764 \pm 0.008$ & $0.764 \pm 0.007$ & $0.766 \pm 0.008$ & $0.763 \pm 0.007$ \\
\hline 8 & $0.644 \pm 0.018$ & $0.646 \pm 0.013$ & $0.644 \pm 0.011$ & $0.642 \pm 0.016$ & $0.647 \pm 0.016$ & $0.647 \pm 0.017$ \\
\hline 9 & $0.858 \pm 0.007$ & $0.855 \pm 0.008$ & $0.850 \pm 0.007$ & $0.847 \pm 0.007$ & $0.846 \pm 0.009$ & $0.846 \pm 0.007$ \\
\hline 10 & $0.663 \pm 0.019$ & $0.656 \pm 0.021$ & $0.650 \pm 0.018$ & $0.651 \pm 0.018$ & $0.648 \pm 0.020$ & $0.651 \pm 0.017$ \\
\hline 11 & $0.741 \pm 0.011$ & $0.748 \pm 0.008$ & $0.747 \pm 0.007$ & $0.743 \pm 0.008$ & $0.739 \pm 0.008$ & $0.736 \pm 0.008$ \\
\hline 12 & $0.806 \pm 0.014$ & $0.799 \pm 0.015$ & $0.801 \pm 0.014$ & $0.804 \pm 0.018$ & $0.805 \pm 0.012$ & $0.804 \pm 0.016$ \\
\hline 13 & $0.917 \pm 0.008$ & $0.920 \pm 0.007$ & $0.920 \pm 0.009$ & $0.920 \pm 0.007$ & $0.921 \pm 0.008$ & $0.920 \pm 0.007$ \\
\hline 14 & $0.871 \pm 0.011$ & $0.870 \pm 0.012$ & $0.869 \pm 0.010$ & $0.872 \pm 0.011$ & $0.875 \pm 0.013$ & $0.875 \pm 0.012$ \\
\hline 15 & $0.969 \pm 0.010$ & $0.963 \pm 0.007$ & $0.960 \pm 0.007$ & $0.957 \pm 0.009$ & $0.955 \pm 0.009$ & $0.950 \pm 0.009$ \\
\hline 16 & $0.623 \pm 0.018$ & $0.666 \pm 0.016$ & $0.706 \pm 0.016$ & $0.734 \pm 0.014$ & $0.747 \pm 0.014$ & $0.760 \pm 0.014$ \\
\hline 17 & $0.835 \pm 0.003$ & $0.836 \pm 0.003$ & $0.837 \pm 0.005$ & $0.837 \pm 0.003$ & $0.837 \pm 0.003$ & $0.837 \pm 0.003$ \\
\hline 18 & $0.780 \pm 0.014$ & $0.780 \pm 0.015$ & $0.779 \pm 0.017$ & $0.776 \pm 0.016$ & $0.775 \pm 0.015$ & $0.767 \pm 0.014$ \\
\hline 19 & $0.935 \pm 0.002$ & $0.938 \pm 0.002$ & $0.938 \pm 0.002$ & $0.939 \pm 0.003$ & $0.941 \pm 0.003$ & $0.941 \pm 0.003$ \\
\hline 20 & $0.935 \pm 0.005$ & $0.938 \pm 0.003$ & $0.937 \pm 0.005$ & $0.938 \pm 0.005$ & $0.937 \pm 0.005$ & $0.937 \pm 0.004$ \\
\hline 21 & $0.867 \pm 0.015$ & $0.871 \pm 0.016$ & $0.871 \pm 0.015$ & $0.868 \pm 0.015$ & $0.871 \pm 0.016$ & $0.872 \pm 0.018$ \\
\hline 22 & $0.766 \pm 0.007$ & $0.766 \pm 0.007$ & $0.765 \pm 0.006$ & $0.764 \pm 0.008$ & $0.765 \pm 0.008$ & $0.766 \pm 0.007$ \\
\hline 23 & $0.746 \pm 0.021$ & $0.745 \pm 0.025$ & $0.752 \pm 0.021$ & $0.750 \pm 0.021$ & $0.753 \pm 0.023$ & $0.755 \pm 0.023$ \\
\hline 24 & $0.783 \pm 0.016$ & $0.779 \pm 0.012$ & $0.780 \pm 0.013$ & $0.780 \pm 0.017$ & $0.780 \pm 0.015$ & $0.775 \pm 0.014$ \\
\hline 25 & $0.698 \pm 0.010$ & $0.714 \pm 0.009$ & $0.710 \pm 0.010$ & $0.715 \pm 0.009$ & $0.715 \pm 0.011$ & $0.714 \pm 0.010$ \\
\hline 26 & $0.773 \pm 0.014$ & $0.776 \pm 0.011$ & $0.779 \pm 0.011$ & $0.777 \pm 0.013$ & $0.775 \pm 0.010$ & $0.774 \pm 0.015$ \\
\hline 27 & $0.969 \pm 0.010$ & $0.971 \pm 0.012$ & $0.971 \pm 0.011$ & $0.970 \pm 0.011$ & $0.971 \pm 0.009$ & $0.972 \pm 0.010$ \\
\hline 28 & $0.595 \pm 0.005$ & $0.595 \pm 0.006$ & $0.597 \pm 0.006$ & $0.596 \pm 0.006$ & $0.596 \pm 0.007$ & $0.596 \pm 0.006$ \\
\hline 29 & $0.551 \pm 0.014$ & $0.549 \pm 0.015$ & $0.548 \pm 0.015$ & $0.547 \pm 0.012$ & $0.548 \pm 0.013$ & $0.547 \pm 0.012$ \\
\hline 30 & $0.598 \pm 0.005$ & $0.597 \pm 0.004$ & $0.596 \pm 0.006$ & $0.596 \pm 0.005$ & $0.596 \pm 0.004$ & $0.595 \pm 0.005$ \\
\hline
\end{tabular}

After we compare the training accuracies of two ELMs on a single data set, Friedman test is used to analyze the difference of training accuracies corresponding to 6 ELMs on 30 data sets. The analyzed object is the experimental results in Tables 2, 4, or 6 .

1) Increasingly order the average training accuracies of 6 ELMs on every data set. Then, the average value of ranks is assigned to the same training accuracy (This depends on the results obtained by previous Wilcoxon signed-ranks test: if the null-hypothesis is accepted, we say the training accuracies of two ELMs are same and thus have the same rank).

2) For 6 ELMs, the average value of ranks $R_{i}(i=1, \cdots, 6)$ on 30 data sets is calculated respectively.

3) Construct the statistic $F_{F}=\frac{(m-1) \chi_{F}^{2}}{m(k-1)-\chi_{F}^{2}}$ based on the null-hypothesis which indicates that all models have the equal training accuracy on all data sets, where $\chi_{F}^{2}=\frac{12 m}{k(k+1)}\left[\sum_{i=1}^{6} R_{i}^{2}-\frac{k(k+1)^{2}}{4}\right]$ is the Friedman statistic, $k$ is the number of learning models, $m$ is the number of data sets. In this study, $k=6$ and $m=30$.

4) The statistic $F_{F}$ obeys F-distribution with $(k-1)$ and $(k-1) \times(m-1)$ freedom degrees. Under significance 
level $\alpha=0.1$, we can get $F_{\alpha}[(k-1),(k-1) \times(m-1)]=F_{0.1}(5,145)=1.887$.

5) If $F_{F}>F_{0.1}(5,145)$, the null-hypothesis can be rejected. That is to say the training accuracies of these 6 ELMs are different on the 30 data sets.

Based on the statistical analysis results with Wilcoxon signed-ranks test and Friedman test, we select the 9 representative data sets and compare the training and testing accuracies of ELMs corresponding to random initialization with different variances for each distribution (Figs. 2 and 3 are training and testing accuracies for different Uniform distributions; Figs. 4 and 5 are training and testing accuracies for different Gamma distributions; and Figs. 6 and 7 are training and testing accuracies for different Normal distributions). From Tables 2-7 and Figs. 2-7, we can find the following experimental observations and further summarize some usefully empirical conclusions.

$\square \mu=0, \sigma=0.05 \square \mu=0, \sigma=0.1 \square \mu=0, \sigma=0.15 \square \mu=0, \sigma=0.2 \quad \boldsymbol{\square} \mu=0, \sigma=0.25 \square \mu=0, \sigma=0.3$

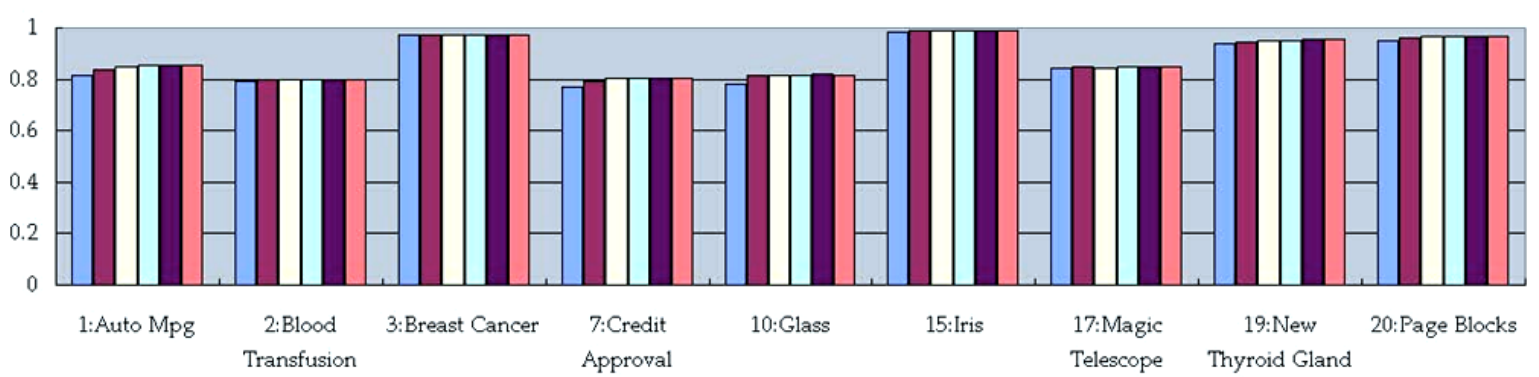

Fig. 6. Training accuracies of 6 ELMs corresponding to 6 Normal distributions on 9 representative data sets.

$\square \mu=0, \sigma=0.05 \square \mu=0, \sigma=0.1 \quad \square \mu=0, \sigma=0.15 \square \mu=0, \sigma=0.2 \quad \boldsymbol{\square} \mu=0, \sigma=0.25 \square \mu=0, \sigma=0.3$

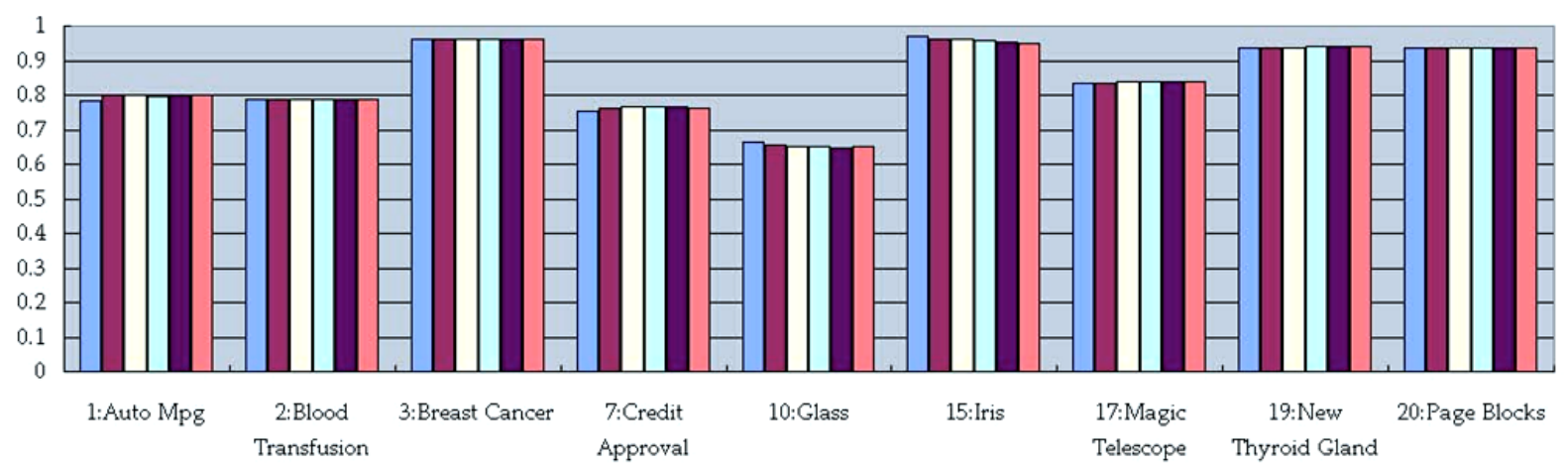

Fig. 7. Testing accuracies of 6 ELMs corresponding to 6 Normal distributions on 9 representative data sets.

1) Uniform and Gamma distributions with the smaller variances usually make ELMs get the higher training and testing accuracies. From Figs. 2 and 3, we can find the training and testing accuracies all obviously (confirmed by above-mentioned statistical analysis) decrease with the increase of variances of Uniform random numbers. The similar observation occurs in Figs. 4 and 5 for Gamma random numbers.

2) Compared with Normal distribution, the variances of Uniform and Gamma distributions have significant impact on the classification performance of ELMs. From Figs. 6 and 7, we can find with the increase of variances of Normal random numbers, the training and testing accuracies don't change significantly. We try to give the analysis to this experimental result. Although $x \in(-\infty,+\infty)$ for Normal distribution $p(\mathrm{x})$, the large percentage (i.e., 99.7\%) of random numbers locate in interval $[\mu-3 \sigma, \mu+3 \sigma]$. From $[-0.15,0.15]$ to $[-0.9,0.9]$, even though the standard deviation of Normal 
distribution is 0.3 , a large number of Normal random numbers in interval $[-0.15,0.15]$ will be selected to initialize the weights and biases.

3) Uniform and Gamma distributions with larger variances could seriously deteriorate the classification capability of ELMs. From Tables 2-5, we can find the training and testing accuracies on Image Segment, Libras Movement, and Vowel Recognition data sets are severely damaged by Uniform and Gamma random numbers with larger variances. However, this phenomenon doesn't occur for Normal random numbers. This is because the random numbers with the larger variances for Uniform and Gamma distributions on Image Segment, Libras Movement and Vowel Recognition data sets make the solution to Moore-Penrose generalized inverse of hidden-layer output matrix H more inaccurate and we can't get $\mathrm{H}^{\dagger}=\left(\mathrm{H}^{\mathrm{T}} \mathrm{H}\right)^{-1} \mathrm{H}^{\mathrm{T}}$ or $\mathrm{H}^{\dagger}=\mathrm{H}^{\mathrm{T}}\left(\mathrm{HH}^{\mathrm{T}}\right)^{-1}$ by using the orthogonal projection method [19].

4) ELMs initialized by Uniform and Gamma distributions with larger variances generally needs more hidden-layer nodes to achieve equivalent classification accuracies with ones having the smaller variances. We conduct an additional experiment to compare the learning capabilities of ELMs corresponding to.

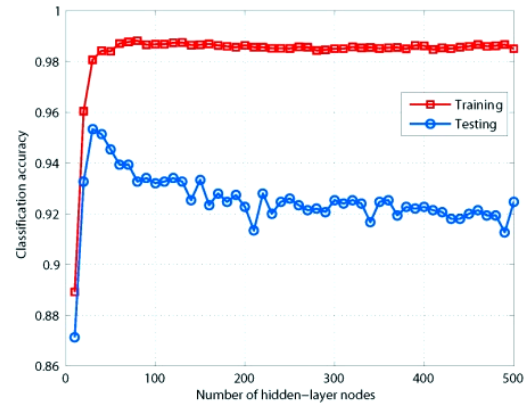

(a) $a=0, b=5$

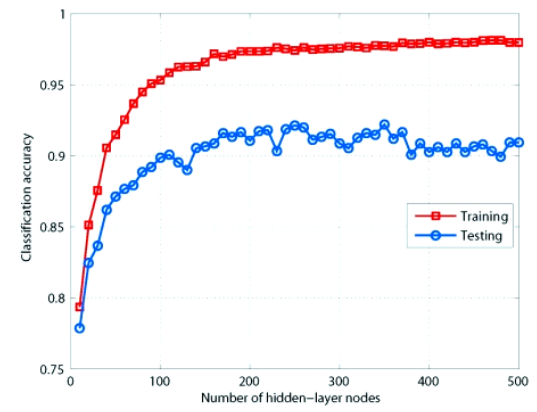

(b) $a=0, b=10$

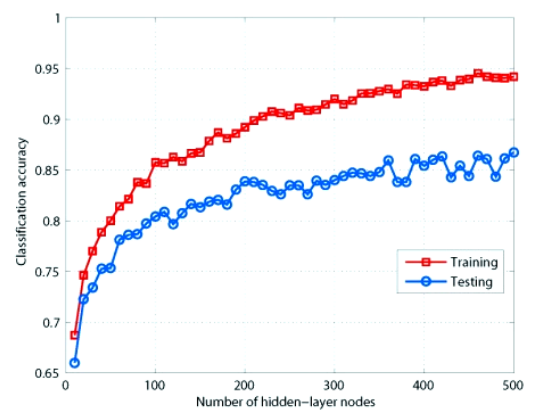

(c) $a=0, b=15$

Fig. 8. Learning capability of ELMs with Uniform initialization in Iris data set.

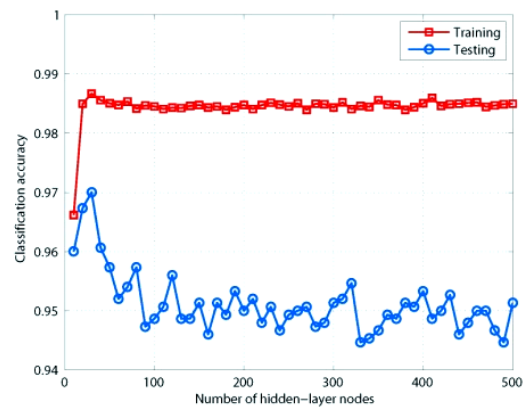

(a) $k=9, \theta=0.1$

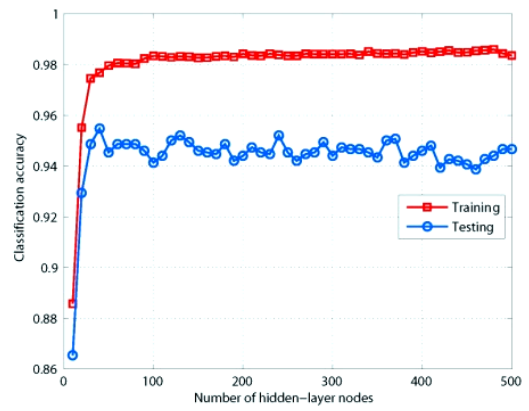

(b) $k=9, \theta=0.3$

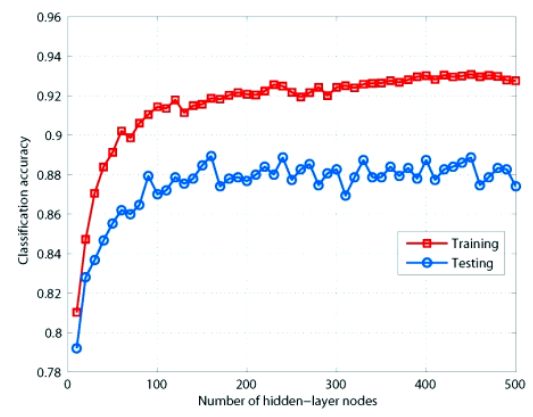

(c) $k=9, \theta=0.5$

Fig. 9. Learning capability of ELMs with Gamma initialization in Iris data set.

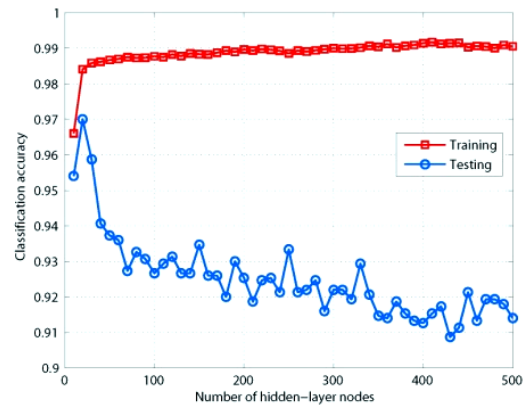

(a) $\mu=0, \sigma=0.05$

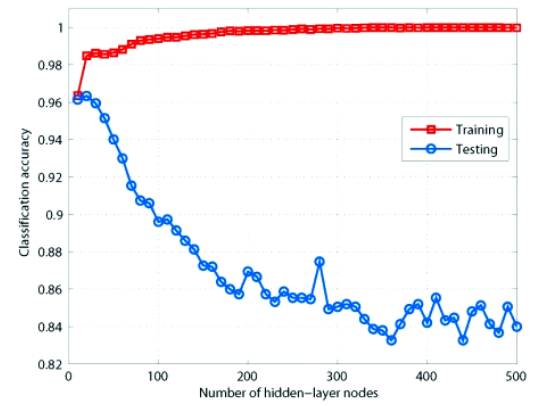

(b) $\mu=0, \sigma=0.1$

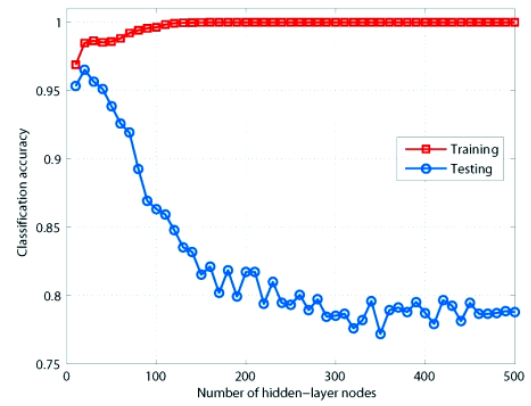

(c) $\mu=0, \sigma=0.15$

Fig. 10. Learning capability of ELMs with Normal initialization in Iris data set. 
random numbers with different variances. Let the number of hidden-layer nodes range from 10 to 300 in step of 10. On Iris data set we plot the learning curves of different ELMs (See Figs. 8-10). For Uniform and Gamma random numbers, we can see that with the increase of variance, ELM needs the more hidden-layer nodes to obtain an acceptable learning capability. This is because a number of hidden-layer nodes will makes orthogonal projection method more effective for random numbers with the larger variance.

5) By comparing Figs. 8-9 with Fig. 10, we can find that Normal distribution is more easily lead to the over-fitting of ELMs. On Normal distribution, we can use the orthogonal projection method to get the more accurate solution to Moore-Penrose generalized inverse of H, i.e., $H^{\dagger}=H^{T}\left(H H^{T}\right)^{-1}$. Furthermore, the following equation can be derived:

$$
H \beta=H\left(H^{\dagger} Y\right)=H\left[H^{T}\left(H H^{T}\right)^{-1} Y\right]=\left(H H^{T}\right)\left(H H^{T}\right)^{-1} Y=Y .
$$

\section{Conclusion}

In this paper, we experimentally validate the impact of variance of random weights and biases on the classification performance of ELMs and give some important and useful enlightenments regarding ELM initialization. By initializing the input-layer weights and hidden-layer biases with random numbers having different variances, we compare the training and testing accuracies of ELMs on 30 UCI data sets. The experimental results and statistical analysis reflect that (1) Uniform and Gamma distributions with the smaller variances usually make ELMs get the higher training and testing accuracies and Uniform and Gamma distributions with the larger variances could seriously deteriorate the classification capability of ELMs; (2) Compared with Normal distribution, the variances of Uniform and Gamma distributions have the significant impact on the classification performance of ELMs; and (3) ELMs initialized by Uniform and Gamma distributions with the larger variances generally needs the more hidden-layer nodes to achieve the equivalent classification accuracies with ones having the smaller variances. In addition, we also discuss the influence of initialization's variance on over-fitting of ELMs.

\section{Acknowledgment}

We thank Editor-in-Chief and anonymous reviewers, whose valuable comments and suggestions help us to improve this paper significantly. The corresponding author, Dr. Yulin He, is supported by China Postdoctoral Science Foundation (2015M572361) and National Natural Science Foundation of China (61503252).

\section{References}

[1] Huang, G. B., Zhu, Q. Y., \& Siew, C. K. (2006). Extreme learning machine: Theory and applications. Neurocomputing, 70(1), 489-501.

[2] Serre, D. (2002). Matrices: Theory and Applications. Springer, New York.

[3] Huang, G. B., Chen, L., \& Siew C. K. (2006). Universal approximation using incremental constructive feedforward networks with random hidden nodes. IEEE Transactions on Neural Networks, 17(4), 879-892.

[4] Huang, G. B., Wang, D. H., \& Lan Y. (2011). Extreme learning Machines: A survey. International Journal of Machine Learning and Cybernetics. 2(2), 107-122.

[5] Zhu, Q. Y., Qin, A., Suganthan, P., \& Huang, G. B. (2005). Evolutionary extreme learning machine. Pattern Recognition, 38(10), 1759-1763. 
[6] Cao, J., Lin, Z., \& Huang, G. B. (2012). Self-adaptive evolutionary extreme learning machine. Neural Processing Letters, 36(3), 285-305.

[7] Matias, T., Souza, F., Araújo, R., \& Antunes, C. H. (2014). Learning of a single-hidden layer feedforward neural network using an optimized extreme learning machine. Neurocomputing, 129, 428-436.

[8] Heeswijk, M. V., \& Miche, Y. (2015). Binary/ternary extreme learning machines. Neurocomputing, 149, 187-197.

[9] Balasundaram, S., \& Gupta, D. (2014) On optimization based extreme learning machine in primal for regression and classification by functional iterative method. International Journal of Machine Learning and Cybernetics.

[10] Deng, W., Zheng, Q., \& Chen, L. (2009). Regularized extreme learning machine. Proceedings of 2009 IEEE Symposium on Computational Intelligence and Data Mining (pp. 389-395).

[11] Horata, P., Chiewchanwattana, S., \& Sunat, K. (2013). Robust extreme learning machine. Neurocomputing, 102, 31-44.

[12] Huang, G. B., Li, M. B., Chen, L., \& Siew, C. K. (2008). Incremental extreme learning machine with fully complex hidden nodes. Neurocomputing, 71(4-6), 576-583.

[13] Miche, Y., Sorjamaa, A., Bas, P., Simula, O., Jutten, C., \& Lendasse, A. (2010). OP-ELM: Optimally pruned extreme learning machine. IEEE Transactions on Neural Networks, 21(1), 158-162.

[14] Wang, X. Z., Shao, Q. Y., Miao, Q., \& Zhai, J. H., (2013). Architecture selection for networks trained with extreme learning machine using localized generalization error model. Neurocomputing, 102, 3-9.

[15] Zhang, K., \& Luo, M. X. (2014). Outlier-robust extreme learning machine for regression problems. Neurocomputing.

[16] He, Y. L., Liu, J. N. K., Wang, X. Z., \& Hu, Y. X. (2012). Optimal bandwidth selection for resubstitution entropy estimation. Applied Mathematics and Computation, 219(8), 3425-3460.

[17] Demšar, J. (2006). Statistical comparisons of classifiers over multiple data sets. Journal of Machine Learning Research, 7(1), 1-30.

[18] Hall, M., Frank, E., Holmes, G., Pfahringer, B., Reutemann, P., \& Witten, I. H. (2009). The WEKA data mining software: An update. SIGKDD Explorations, 11(1), 10-18.

[19] Huang, G. B., Zhou, H., Ding, X., \& Zhang, R. (2012). Extreme learning machine for regression and multiclass classification. IEEE Transactions on Systems, Man, and Cybernetics, Part B: Cybernetics, 42(2), 513-529.

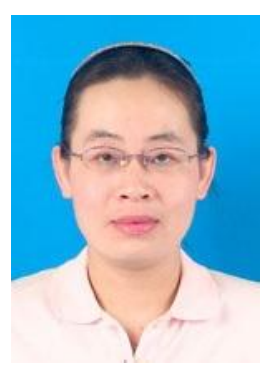

Tao Xiao received her bachelor's degree in mathematics and mathematics education from Hebei Normal University in June 2003 and received her master's degree in applied mathematics from Hebei University in June 2009. Since July 2003, she has been a lecturer of College of Science in Hebei Agricultural University, engaging in mathematics teaching. Her research interests include fuzzy mathematics and uncertain information processing.

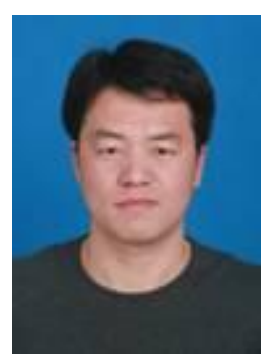

Xu Zhou received his bachelor's degree in mathematics and application from Agricultural University of Hebei, Baoding, China, in June 2010. He received his master degree in application mathematics from Hebei University of Hebei, Baoding, China, in June 2013. He is currently a mathematics teacher in Agricultural University of Hebei, Baoding, China. His research interests include artificial neural networks, machine learning, extreme learning machine, and support vector machine. 


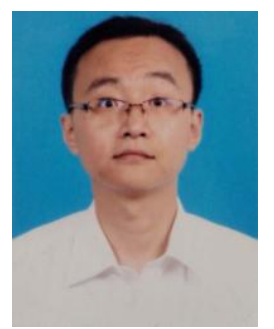

Yu Lin He received both his master degrees in computer science and Ph.D. degree in optical engineering from Hebei University in June 2009 and June 2014, respectively. He is currently a post-doctoral fellow in College of Computer Science and Software Engineering, Shenzhen University, Shenzhen, Guangdong, China. From February 2011 to January 2012 and from August 2013 to August 2014, he has served as a research assistant in the Department of Computing, Hong Kong Polytechnic University, Hong Kong. His research interests include bayesian network, artificial neural networks, evolutionary optimization, probability density estimation, and approximate reasoning. He is the assistant of editor-in-chief of International Journal of Machine Learning and Cybernetics (Science Citation Index Expanded).

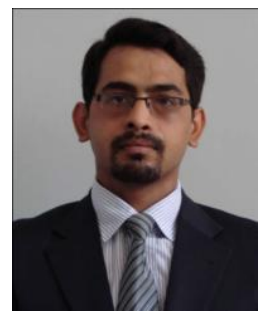

Rana Aamir Raza Ashfaq received his master degree in computer science from Blekinge Tekniska Hgskola (BTH), Sweden. He also received his bachelor and master degrees in computer science from Bahauddin Zakariya University, Multan, Pakistan. Since 2010 he is working as an assistant professor in the Department of Computer Science, Bahauddin Zakariya University, Multan, Pakistan. He is currently a Ph.D. student in College of Computer Science and Software Engineering, Shenzhen University, Shenzhen, Guangdong, China. His main research interests include machine learning and big data mining. 